\title{
New zinc ion parameters suitable for classical MD simulations of zinc metallo-peptidases
}

Antonija Tomića,c, Gordan Horvat ${ }^{b}$, Michael Ramek ${ }^{c}$, Dejan Agićd, Hrvoje Brkiće,f, and Sanja Tomića*

\footnotetext{
a Division of Organic Chemistry and Biochemistry, Ruđer Bošković Institute, Bijenička 54, 10000 Zagreb, Croatia.

${ }^{b}$ Department of Chemistry, Faculty of Science, University of Zagreb, Horvatovac 102A, 10000 Zagreb, Croatia.

c Institute of Physical and Theoretical Chemistry, Graz University of Technology, Stremayrgasse 9, 8010 Graz, Austria.

d Faculty of Agrobiotechnical Sciences Osijek, Josip Juraj Strossmayer University of Osijek, Petra Svačića 1d, 31 000 Osijek, Croatia.

e Faculty of Medicine, Josip Juraj Strossmayer University of Osijek, J. Huttlera 4, 31000

Osijek, Croatia.
}

${ }^{f}$ Faculty of dental medicine and health, Josip Juraj Strossmayer University of Osijek, Crkvena 21, 31000 Osijek, Croatia.

*sanja.tomic@irb.hr 


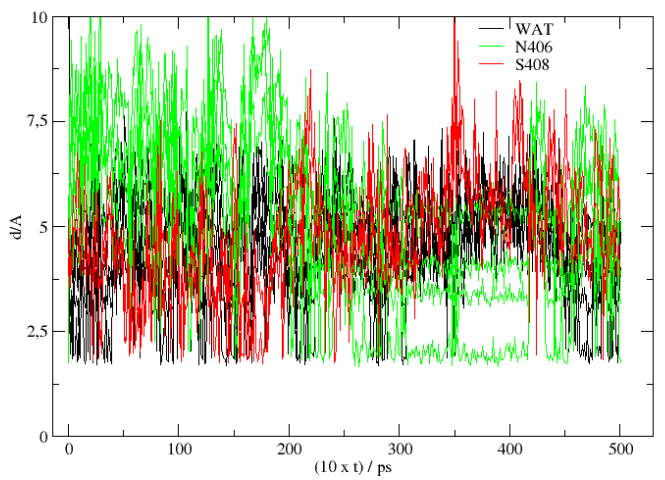

a)

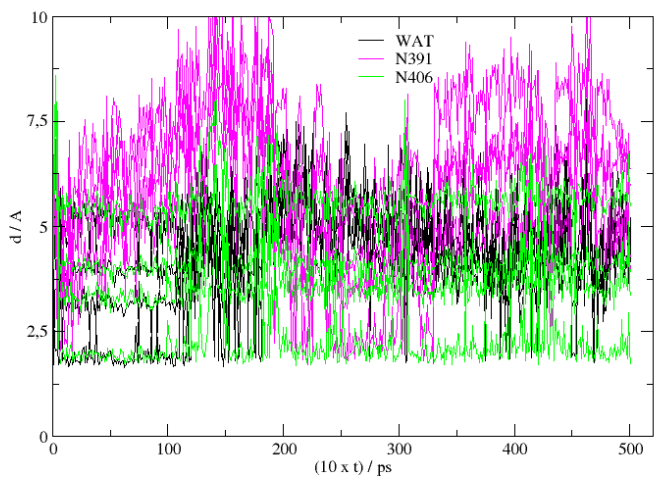

b)

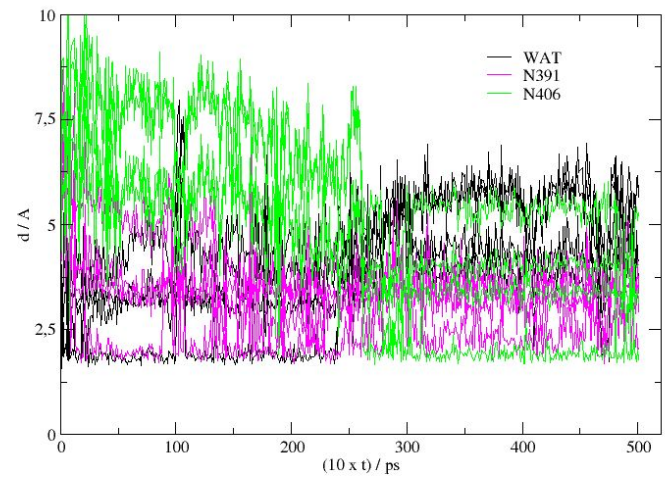

c)

Figure S1. Distances between selected residues polar (hydroxide or amide) hydrogen atoms to the both E451 carboxyl oxygens during MD simulations utilizing the polarizable Drude-2013 force field: a) oDPP III (3FVY), b) cDPP III (5EGY) and c) CDPP III - L-en (QMMM). For clarity distances between specific atoms are not indicated, only the residue to which it is calculated.
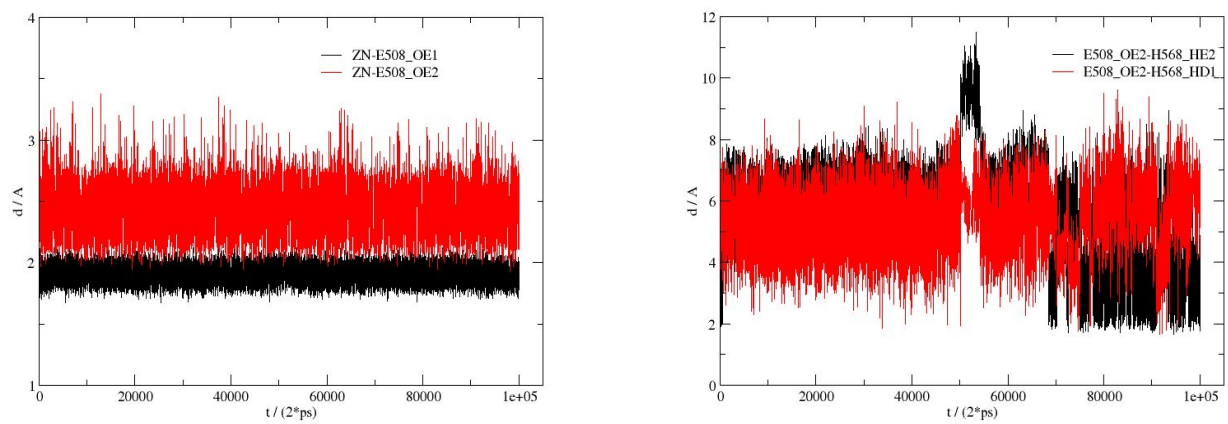

a) 

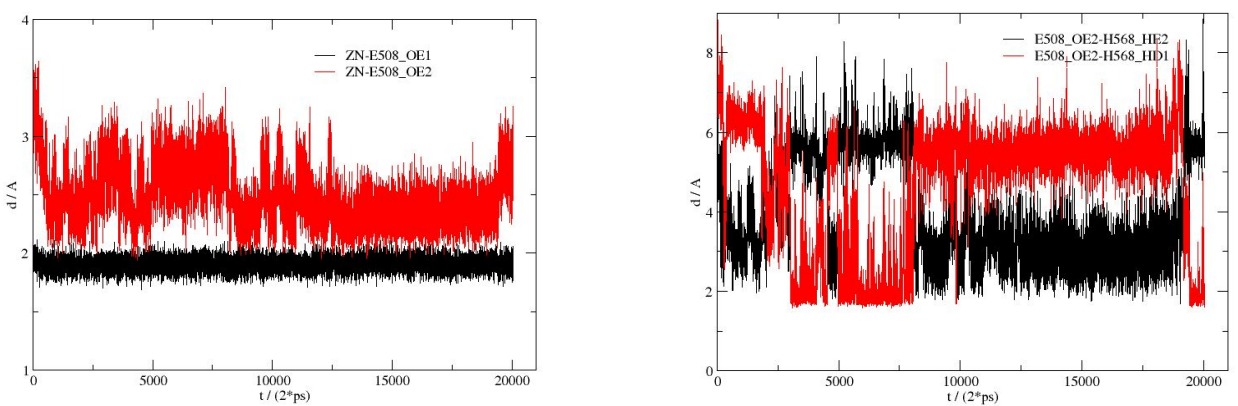

b)
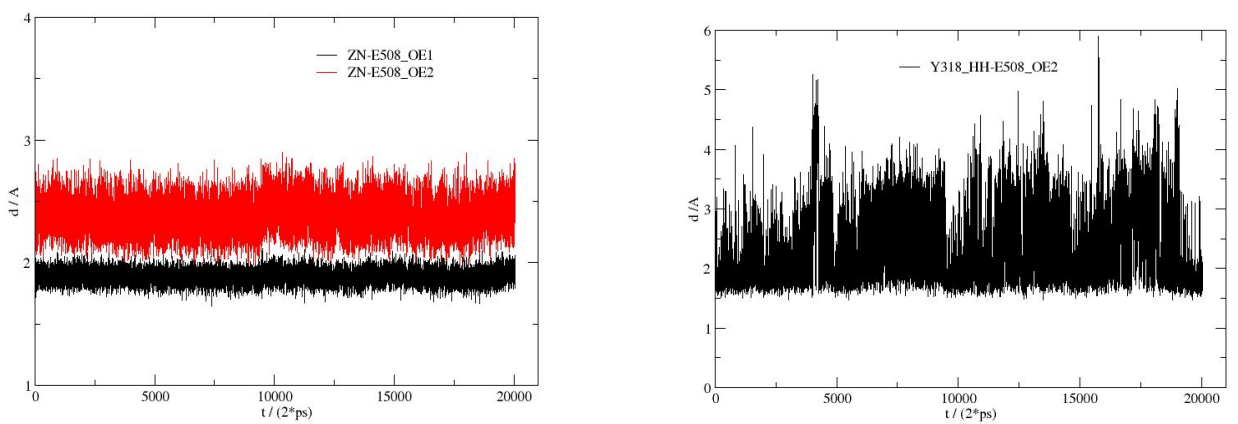

c)
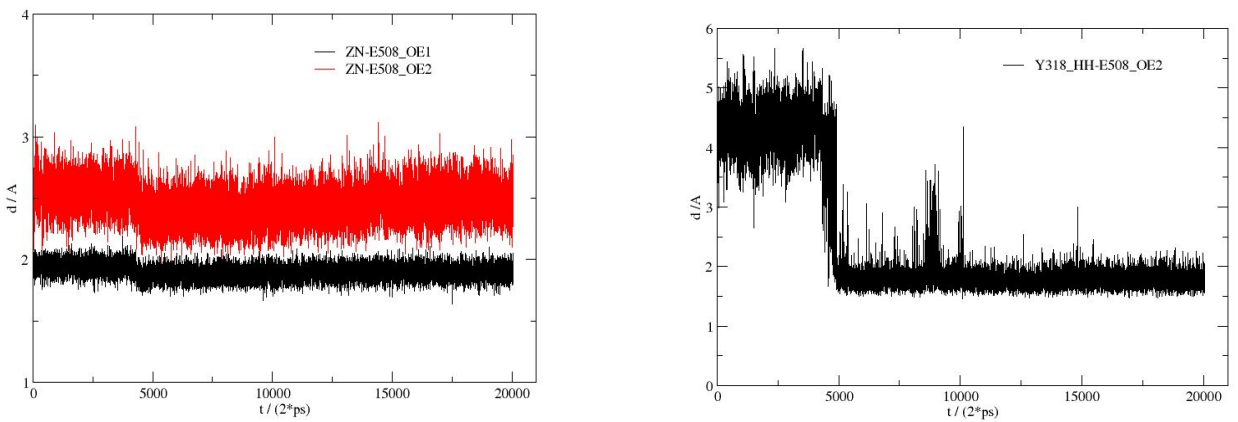

d)

Figure S2. Selected distance along MD simulations utilizing 4-ligand model parameters for the zinc coordination shell: a) oDPP III (3FVY), b) cDPP III (5EGY), c) cDPP III - L-en (5E3A) and d) cDPP III - L-en (QMMM) systems. 

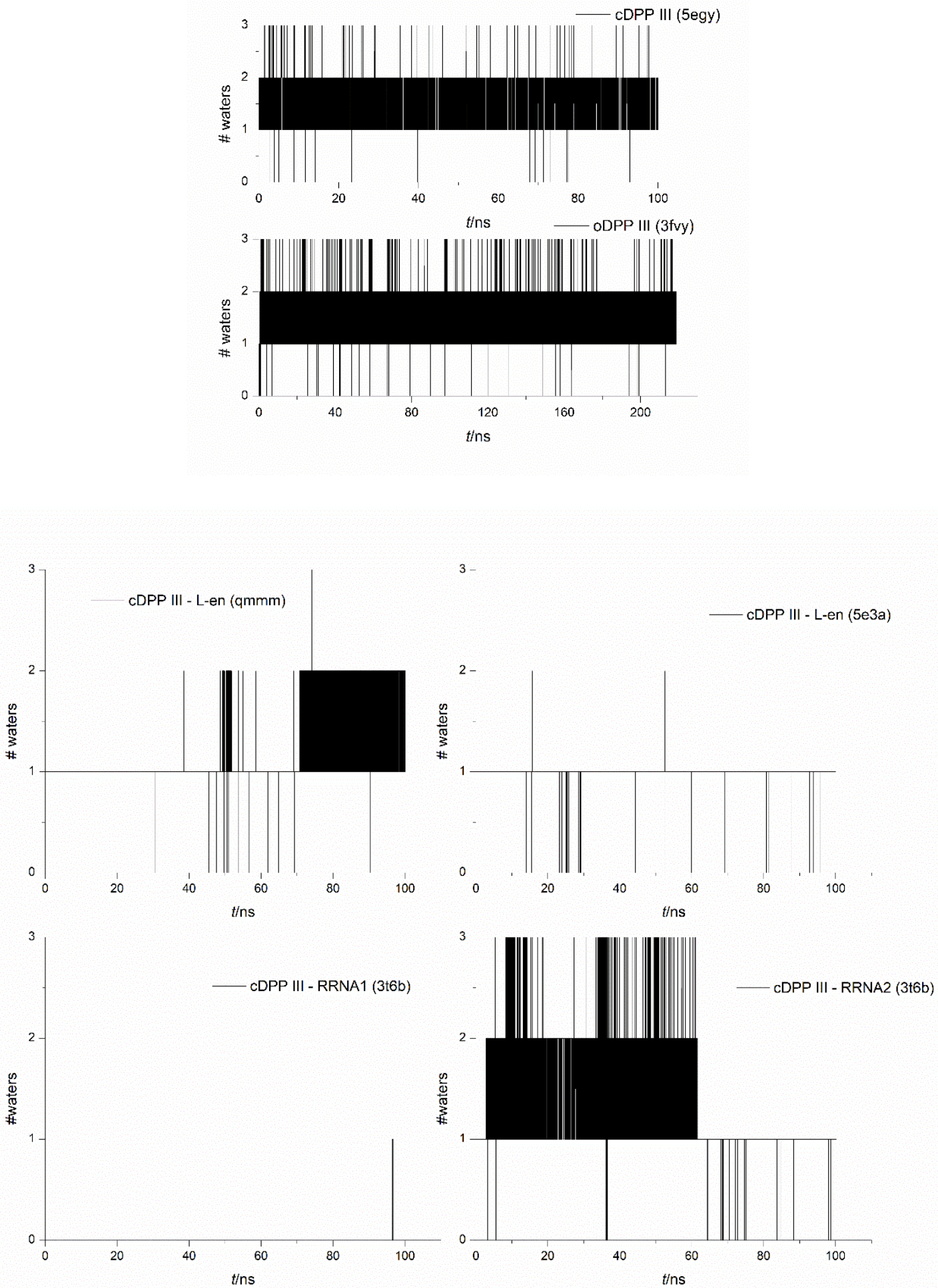

Figure S3. The number of water molecules (\# waters) found within $2.5 \AA$ from the zinc ion during the MD simulations utilizing the 4-ligand-extended model parameters. 

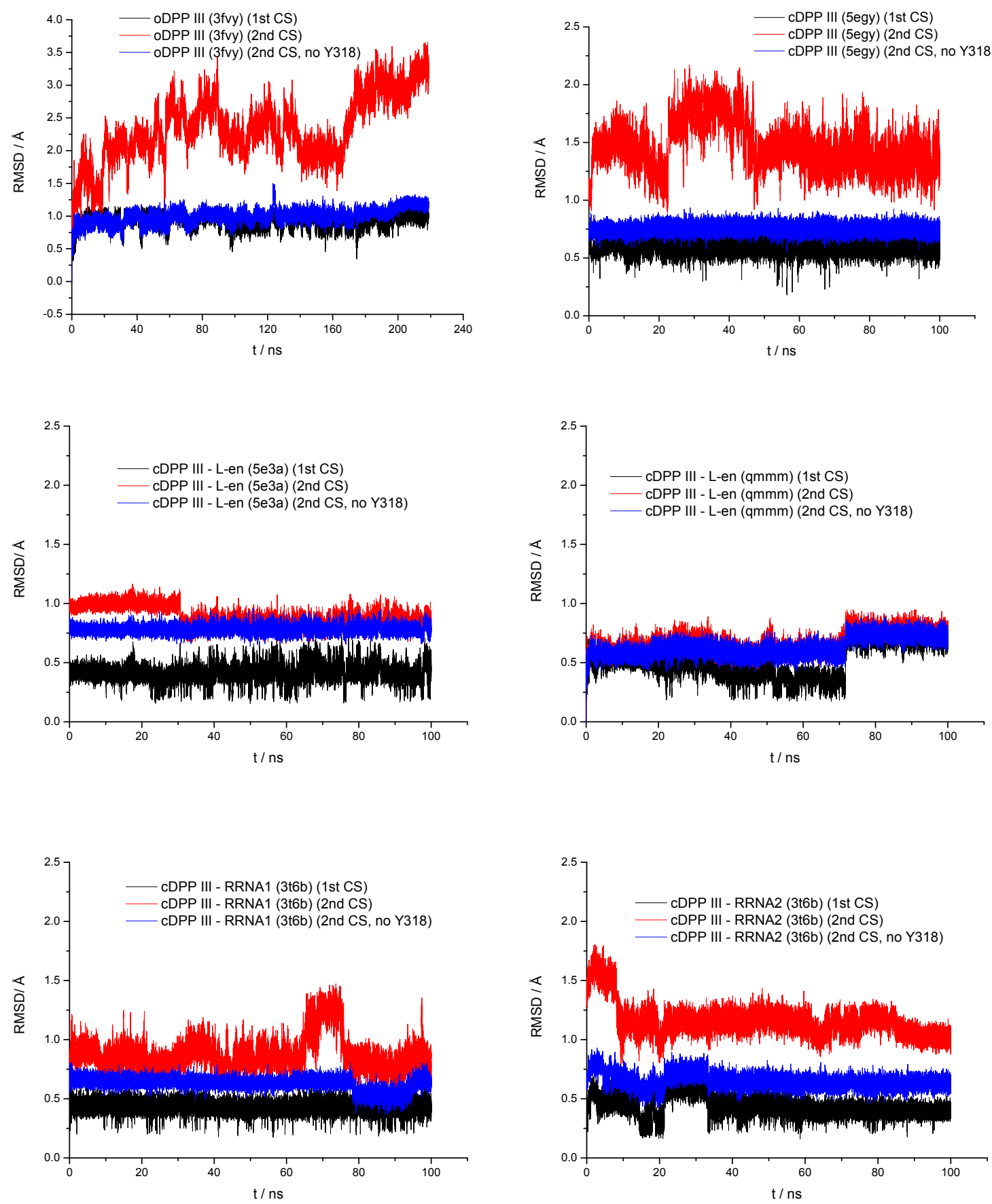

Figure S4. RMSD values of the zinc $1^{\text {st }}$ (the heavy atoms of the $\mathrm{H} 450, \mathrm{H} 455$ and E508 residues, line colored black) and $2^{\text {nd }}$ (the heavy atoms of the Y318, H450, E451, H455, E507, E508, E512 residues, line colored red) coordination sphere (CS) monitored along the MD trajectory for unbound (open, ODPP III, and closed, cDPP III) and bound (Leu-enkephalin, L-en, and Arg-Arg-2-naphthylamide, RRNA) human DPP III structures. RMSD profiles were calculated even when Tyr318 was not considered as a part of the zinc $2^{\text {nd }} \mathrm{CS}$ (blue line). RMSD profiles for each trajectory were calculated against QMMM optimized CDPP III - L-en complex structures (in a case of the closed DPP III, bound and unbound) or relevant PDB (3FVY in a case of oDPP III) structure. 

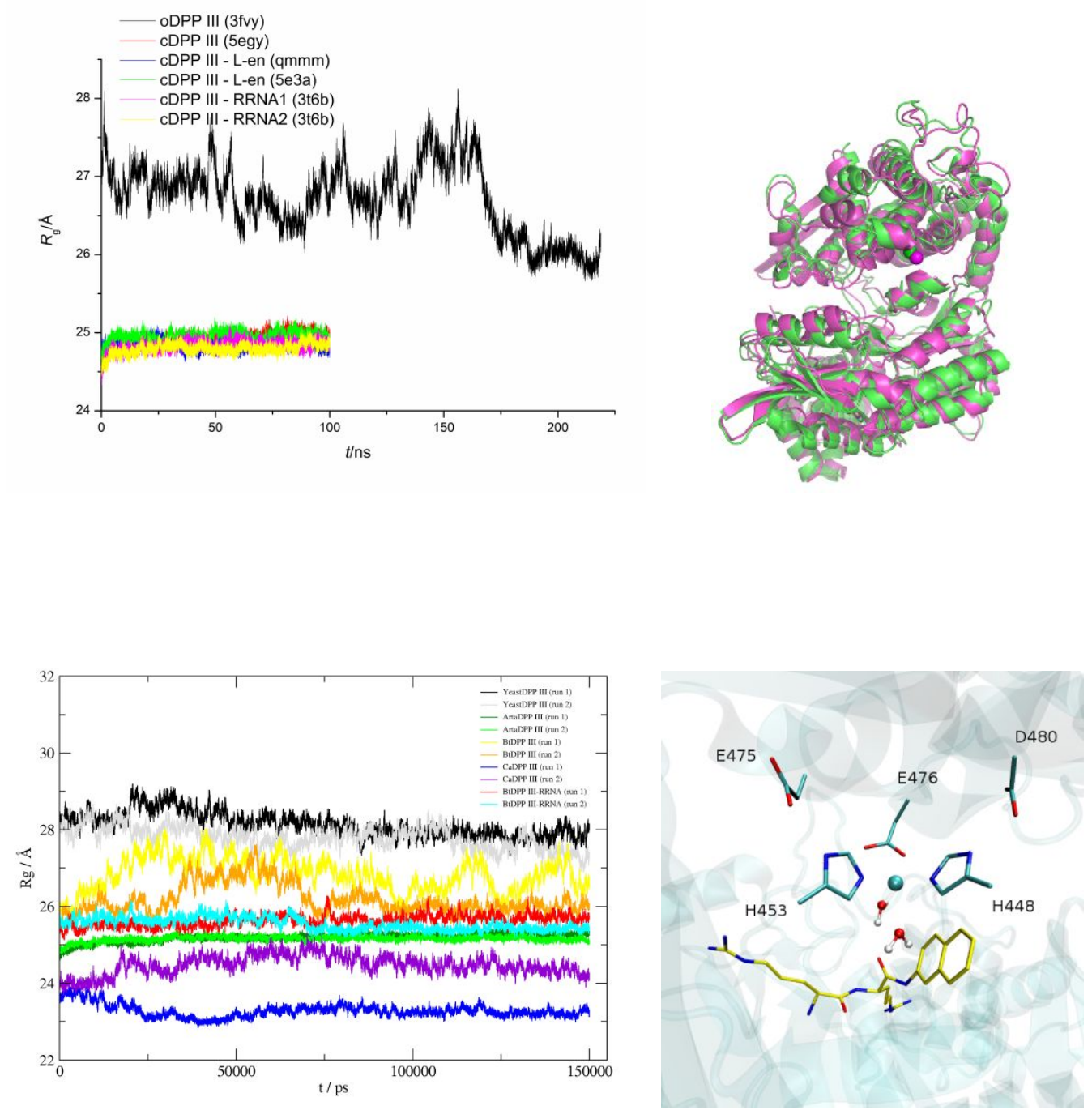

Figure S5. Up-left, the protein backbone radius of gyration $\left(R_{\mathrm{g}}\right)$ profiles obtained during $\mathrm{MD}$ simulations of open (ODPP III) and closed (CDPP III) DPP III, either ligand free or bound (Leu-enkephalin, L-en, and Arg-Arg-2-naphthylamide, RRNA), using the 4-ligand-extended model of the zinc coordination sphere. Residues from protein "flexible loop" (residues 463 to 489) were omitted from the calculation. Up-right, aligned structures of CDPP III obtained by MD simulations utilizing 12-6 Ltype nonbonded (magenta, $R_{\mathrm{g}, \min }=25.34 \AA$ ) and 4-ligand-extended model parameters (green, $R_{\mathrm{g}, \min }=25.66 \AA$ ) for the zinc ion (shown as sphere).

Down-left, the protein backbone radius of gyration $\left(R_{\mathrm{g}}\right)$ profiles obtained during 150 ns of MD simulations of ligand-free DPP III orthologues: y.DPP III, At.DPP III, Ca.DPP III, Bt.DPP III and the Bt.DPP III RRNA complex. Down-right the first and the second zinc ion coordination shell and substrate (ArgArg-2-naphthylamide, RRNA) position in the optimised structure of BtDPP III-RRNA complex obtained after 150 ns of MD simulations. Carbon atoms in supstrat molecule are colored yellow. Backbone and hydrgen atoms are not shown for clarity. 

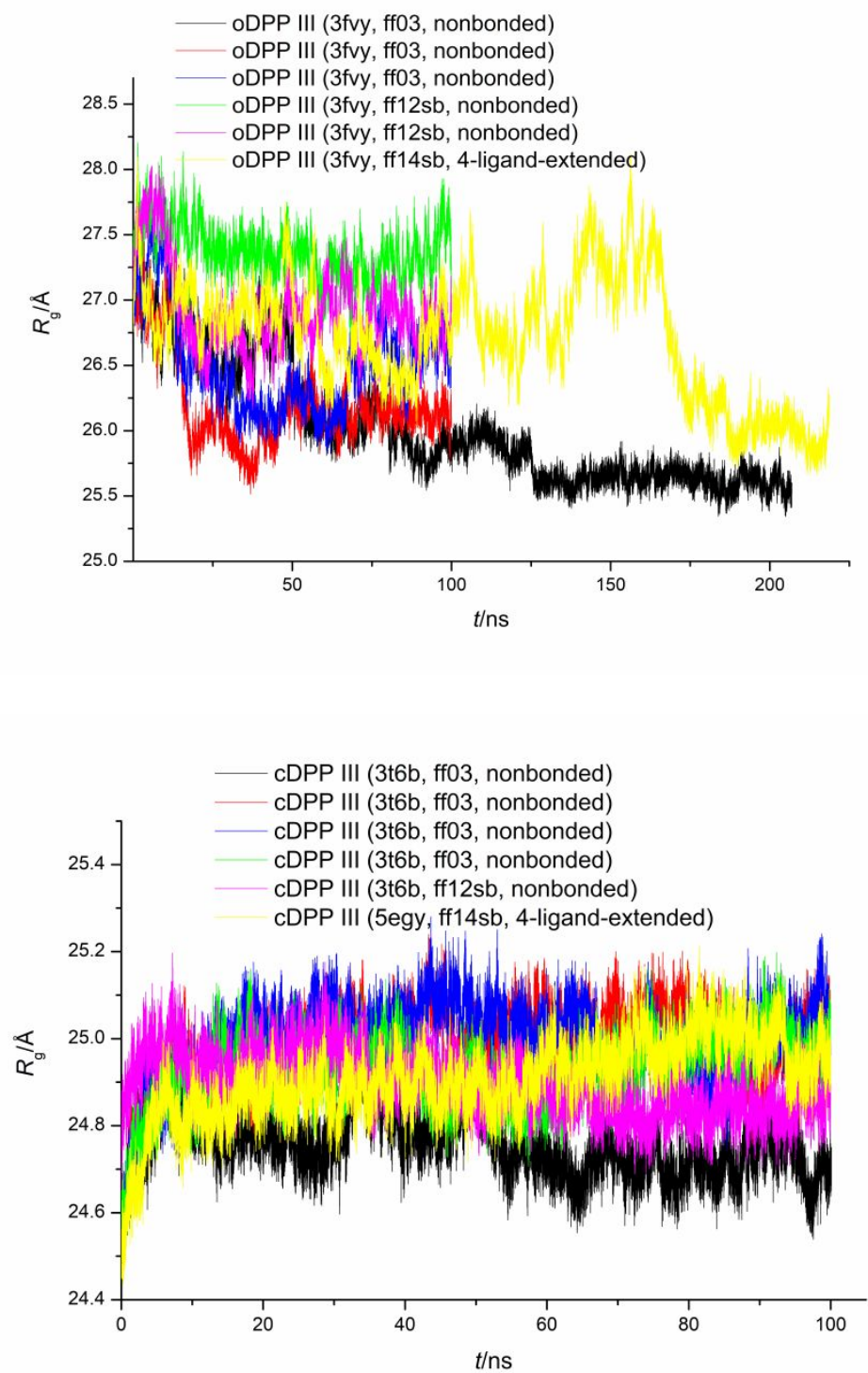

Figure S6. Radius of gyration $\left(R_{\mathrm{g}}\right)$ profiles obtained during MD simulations started from the open (oDPP III, top) and closed (cDPP III, bottom) ligand-free DPP III structure. For each simulation initial structure, force field and zinc ion parameters are indicated in paranthesis. Simulations utilizing nonbonded parameters are from previous work..$^{24,37}$ 

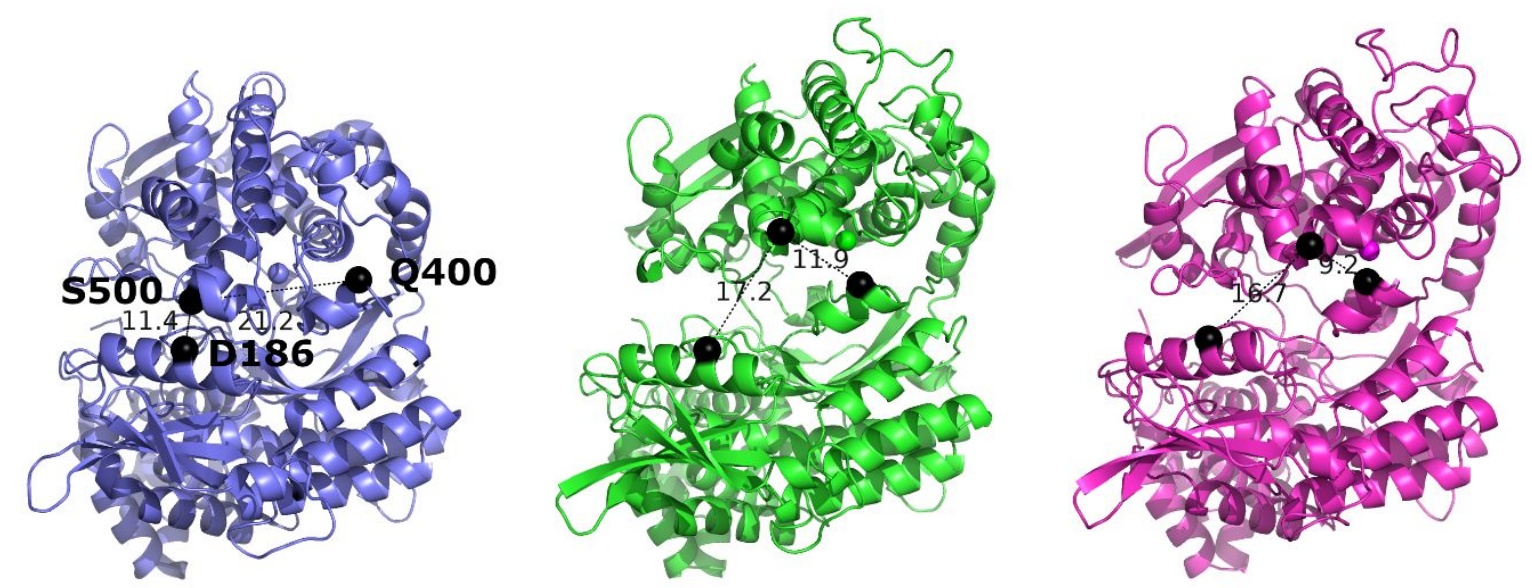

Figure S7. Cartoon representation of closed h.DPP III structures determined by X-ray diffraction (blue, PDB code: $5 E G Y$ ), and that obtained by oDPP III (3FVY) structure MD simulations utilizing the 4-ligandextended model parameters for the zinc coordination shell (green, $R_{\mathrm{g}, \min }=25.66 \AA$ at $211916 \mathrm{~ns}$ ) and 12-6 L-type nonbonded parameters ${ }^{37}$ for the zinc ion (magenta, $R_{\mathrm{g}, \min }=25.34 \AA$ at $205024 \mathrm{~ns}$ ). C $\alpha$ atoms of residues D186, Q400 and S500 are shown as black spheres, and $d_{1}$ (D186-S500) and $d_{2}$ (Q400$\mathrm{S500}) \mathrm{C} \alpha$ distances are indicated by the black dashed lines. The zinc ion is show as a sphere and colored as the corresponding protein structure.
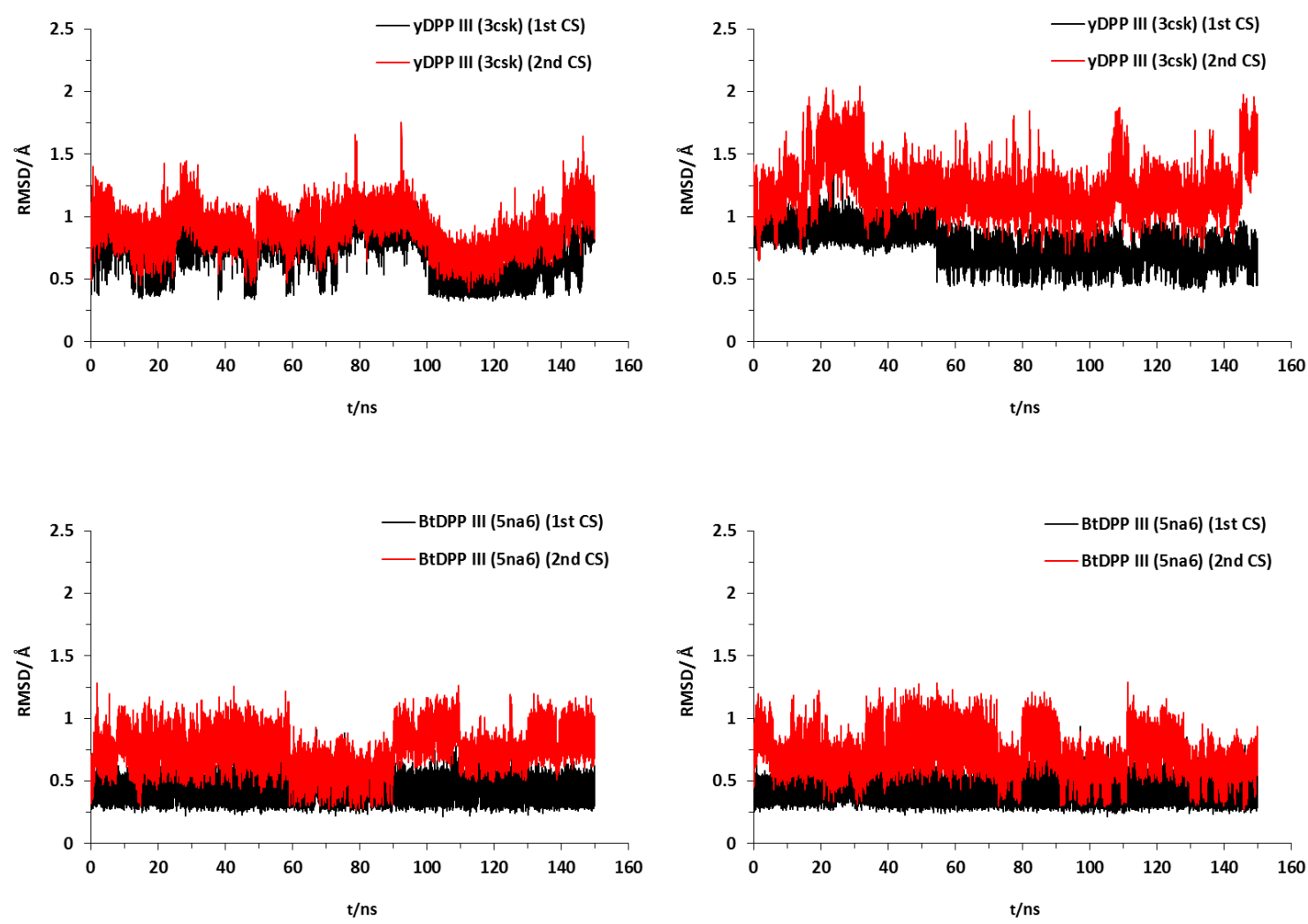

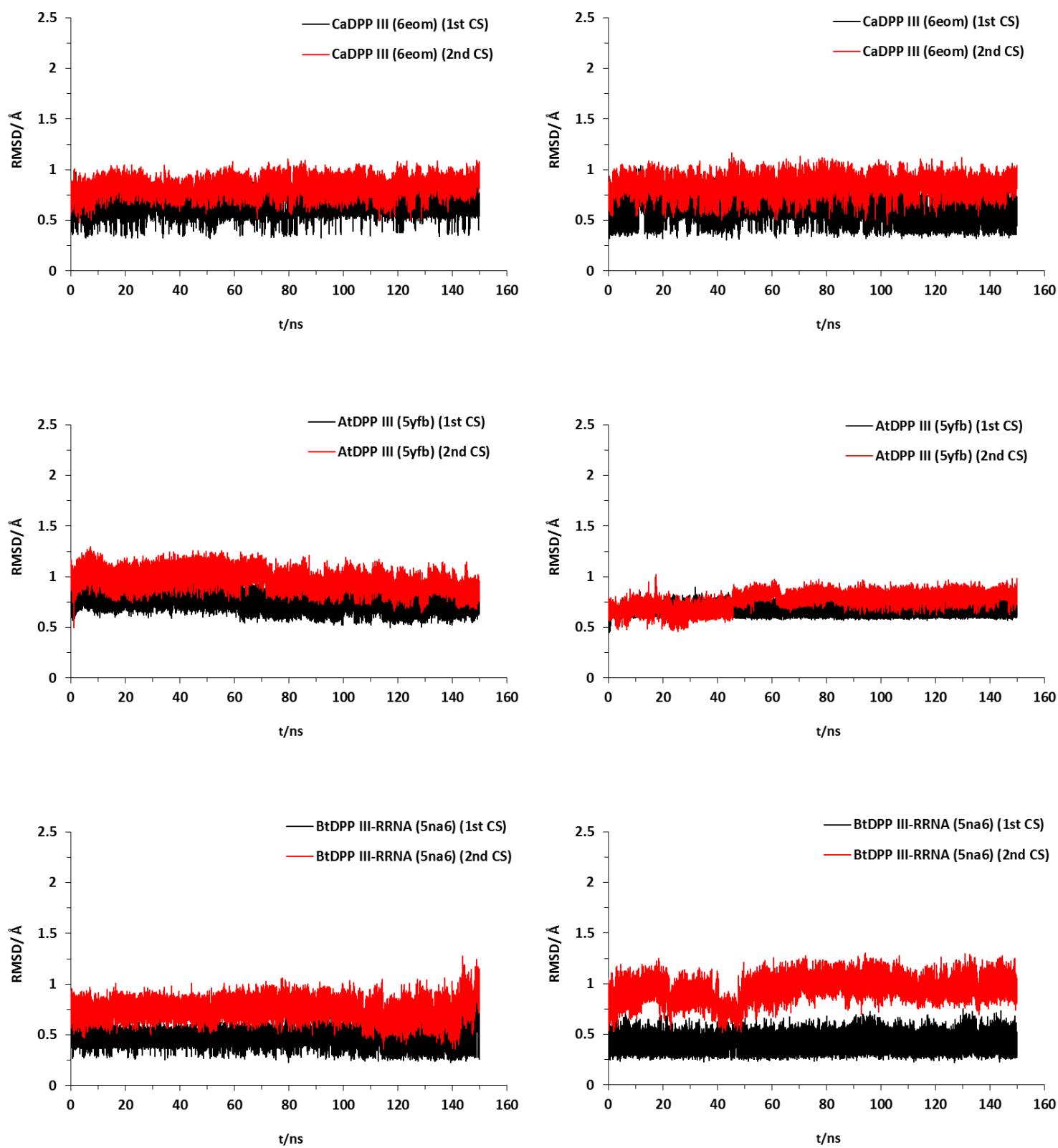

Figure S8. RMSD values of the zinc first and second coordination sphere monitored along the MD trajectory for unbound (yDPP III, BtDPP III, CaDPP III and AtDPP III) and bound (BtDPP III - RRNA) structures of the DPP III orthologues. The first coordination sphere (1st CS) consists of heavy atoms of the residues: $\mathrm{H} 460, \mathrm{H} 465$ and $\mathrm{E} 517$ in the YDPP III; $\mathrm{H} 448, \mathrm{H} 453$ and $\mathrm{E} 476$ in the BtDPP III and BtDPP III - RRNA; H379, H383 and E412 in the CaDPP III while H443, H448 and E501 in the AtDPP III. The second coordination sphere (2nd CS) consists of heavy atoms of the residues: H460, E461, H465, E516, E517 and E520 in the YDPP III; H448, E449, H453, E475, E476 and D480 in the BtDPP III and BtDPP III - RRNA; H379, E380, H383, E411, E412 and D416 in the CaDPP III while H443, E444, H448, E500, E501 and E505 in the AtDPP III structure. The left side marks the first run, and the right side is the second run. RMSD profiles for each trajectory were calculated against relevant crystal structure. 
(4ZR5) 1st CS

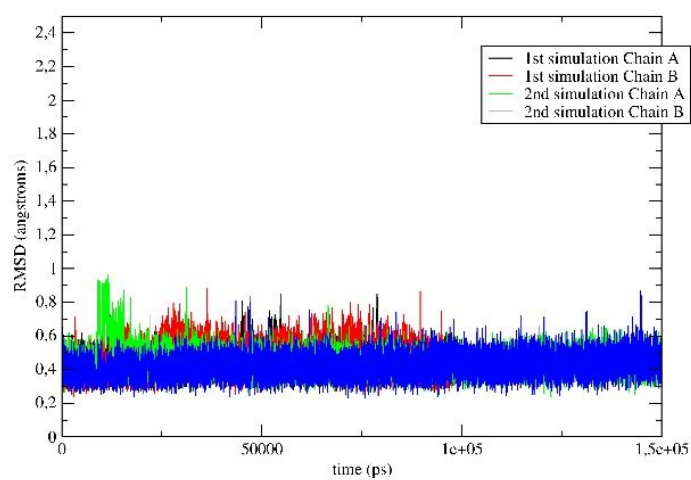

(4XN4) 1st CS

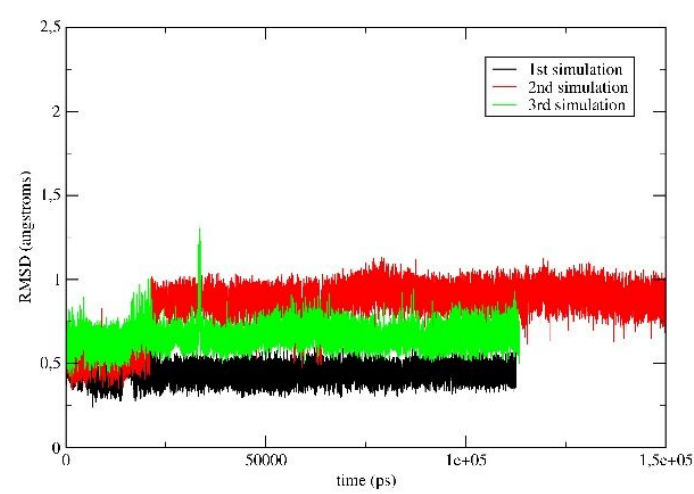

(5A3Y) 1st CS

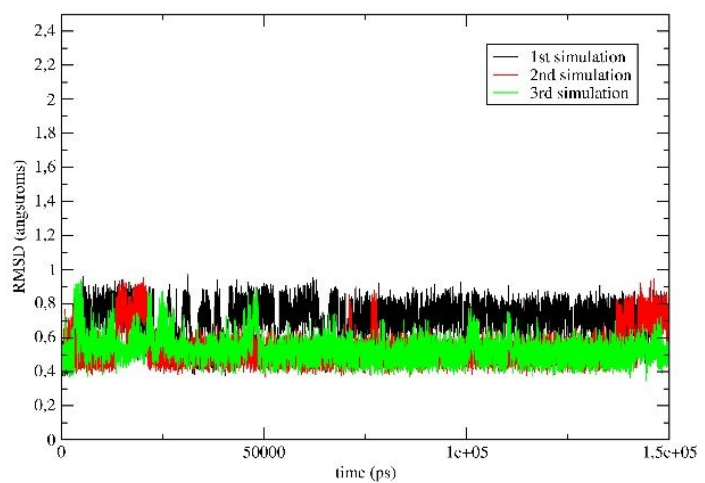

(4ZR5) 2nd CS

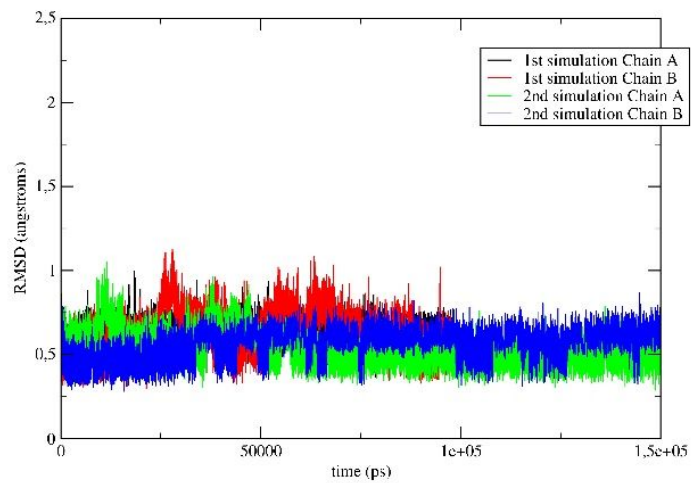

(4XN4) 2nd CS

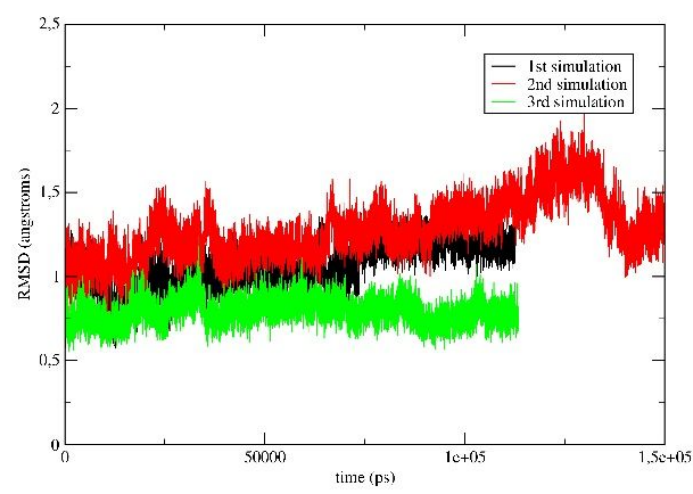

(5A3Y) 2nd CS

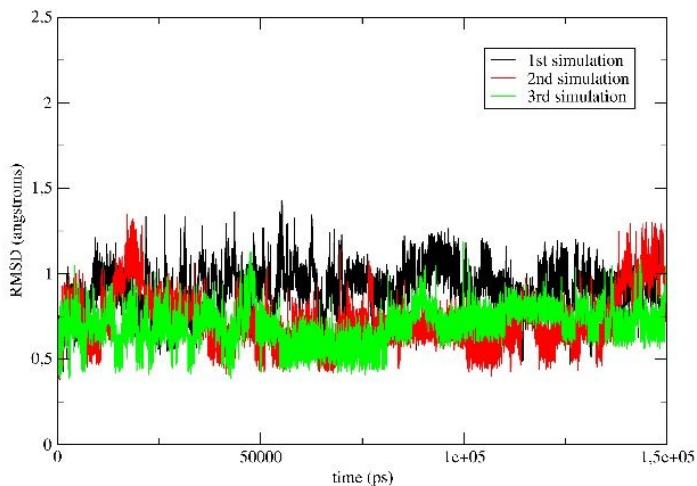

Figure S9. RMSD values of the zinc first $\left(1^{\text {st }}\right)$ and second $\left(2^{\text {nd }}\right)$ coordination spheres $(C S)$ for neprilysine (4ZR5, two figures at the top), aminopeptidase $N(4 \times n 4$, two figures in the middle) and thermolysine (5A3Y, two figures at the bottom). The first zinc ion coordination sphere (left figures represent $1^{\text {st }} \mathrm{CS}$ ) consists of heavy atoms of the residues: H142, H146 and E166 in the thermolysin; H297, H301 and E320 in the aminopeptidase $\mathrm{N}$ while H584, H588 and E647 in the neprilysin. The second zinc ion coordination sphere (right figures represent $2^{\text {nd }}$ CS) consists of heavy atoms of: H142, H146, E166, D170, D150, G162, N165 and D138 in the thermolysin; H297, H301, E320, D327, V324, T304, L316, N306 and K319 in the aminopeptidase N while H584, H588, E647, D591, D651, M580 and T644 in the neprilysin. RMSD profiles were calculated against relevant crystal structures. 
Table S1. Distances $(\AA \AA)$ between the active site zinc ion and its ligands in structures available in PDB. $\mathrm{CN}=$ coordination number.

\section{Human DPPs III with the zinc ion in the active site}

\begin{tabular}{|c|c|c|c|c|c|c|c|c|c|c|}
\hline PDB & organism & ligand & $\mathrm{Zn}-\mathrm{H}$ & $\mathrm{Zn}-\mathrm{H}$ & $\begin{array}{l}\text { Zn-E(O) } \\
\text { closer }\end{array}$ & $\begin{array}{l}\text { Zn- } \\
E(0)\end{array}$ & $\begin{array}{l}\text { Zn-ligand } \\
\text { closest }\end{array}$ & $\begin{array}{l}\text { Zn- } \\
\text { ligand }\end{array}$ & $\mathrm{CN}$ & $\begin{array}{l}\text { Resolu } \\
\text {-tion/Å }\end{array}$ \\
\hline $5 e 33$ & Human & $\begin{array}{l}\text { (met-enkephalin) } \\
\text { water }\end{array}$ & 1.98 & 2.07 & 2.13 & 3.07 & 2.51 & (3.68) & 4 & 2.4 \\
\hline $5 e 3 a$ & Human & leu--enkephalin & 2.00 & 2.01 & 2.01 & 2.82 & & & 3 & 2.1 \\
\hline $5 e 3 c$ & Human & IVYPW & 2.05 & 2.07 & 2.06 & 2.94 & 2.08 & & 4 & 2.8 \\
\hline 5egy & Human & water & 2.04 & 2.07 & 2.10 & 2.96 & & & 3 & 2.7 \\
\hline $53 \mathrm{hh}$ & Human & endomorphin-2 & 1.96 & 2.07 & 1.96 & 3.65 & 2.03 & & 4 & 2.4 \\
\hline $3 f v y$ & Human & water & 2.06 & 2.04 & 2.05 & 3.13 & 2.39 & 2.63 & 4 & 1.9 \\
\hline
\end{tabular}

Other DPP III orthologues

\begin{tabular}{|c|c|c|c|c|c|c|c|c|c|c|}
\hline PDB & organism & ligand & $\mathrm{Zn}-\mathrm{H}$ & $\mathrm{Zn}-\mathrm{H}$ & $\begin{array}{l}\mathrm{Zn}-\mathrm{E}(\mathrm{O}) \\
\text { closer }\end{array}$ & $\begin{array}{l}\mathrm{Zn-} \\
\mathrm{E}(0)\end{array}$ & $\begin{array}{l}\text { Zn-ligand } \\
\text { closest }\end{array}$ & $\begin{array}{l}\mathrm{Zn-} \\
\text { ligand }\end{array}$ & $\mathrm{CN}$ & $\begin{array}{l}\text { Resolu } \\
\text {-tion/Å }\end{array}$ \\
\hline 5 na6 & $\begin{array}{l}\text { Bacteroides } \\
\text { thetaiotaomicron }\end{array}$ & $\begin{array}{l}\text { 2-amino-2- } \\
\text { hydroxymethyl- } \\
\text { propane-1.3-diol }\end{array}$ & 2.07 & 2.09 & 2.18 & 3.21 & 2.12 & $\begin{array}{l}2.15 \\
2.4\end{array}$ & 6 & 1.9 \\
\hline 5 na7 & $\begin{array}{l}\text { Bacteroides } \\
\text { thetaiotaomicron }\end{array}$ & water & 2.04 & 2.05 & 1.94 & 3.69 & 2.13 & 2.11 & 5 & 2.4 \\
\hline 6eom & Caldithrix abyssi & water & 2.03 & 2.03 & 2.00 & 2.01 & 2.08 & & 5 & 2.1 \\
\hline $\begin{array}{l}5 y f b \\
\text { (chain a) }\end{array}$ & $\begin{array}{l}\text { Armillaria } \\
\text { tabescens }\end{array}$ & water & 2.03 & 1.94 & 1.98 & 2.69 & 2.03 & 2.48 & 5 & 2.2 \\
\hline $\begin{array}{l}5 y f b \\
\text { (chain b) }\end{array}$ & $\begin{array}{l}\text { Armillaria } \\
\text { tabescens }\end{array}$ & water & 2.04 & 1.98 & 1.99 & 2.81 & 2.33 & & 4 & 2.2 \\
\hline 3 csk & $\begin{array}{l}\text { Saccharomyces } \\
\text { cerevisiae }\end{array}$ & water & 2.07 & 2.06 & 2.07 & 2.97 & 2.21 & & 4 & 1.9 \\
\hline
\end{tabular}

\section{Aminopeptidase $\mathbf{N}$}

\begin{tabular}{|c|c|c|c|c|c|c|c|c|c|c|}
\hline PDB & organism & ligand & $\mathrm{Zn}-\mathrm{H}$ & $\mathrm{Zn}-\mathrm{H}$ & $\begin{array}{l}\text { Zn-E(O) } \\
\text { closer }\end{array}$ & $\begin{array}{l}\mathrm{Zn-} \\
\mathrm{E}(0)\end{array}$ & $\begin{array}{l}\text { Zn-ligand } \\
\text { closest }\end{array}$ & $\begin{array}{l}\mathrm{Zn}- \\
\text { ligand }\end{array}$ & $\mathrm{CN}$ & $\begin{array}{l}\text { Resolu } \\
\text {-tion/Å }\end{array}$ \\
\hline $2 \mathrm{dq} 6$ & $\begin{array}{l}\text { Escherichia } \\
\text { coli }\end{array}$ & water & 2.06 & 2.02 & 2.02 & 2.92 & 2.32 & & 4 & 1.5 \\
\hline $2 \mathrm{dqm}$ & $\begin{array}{l}\text { Escherichia } \\
\text { coli }\end{array}$ & $\begin{array}{l}\text { 2-(3-amino-2-hydroxy-4 - } \\
\text { phenyl-butyrylamino)-4- } \\
\text { methyl- pentanoic acid }\end{array}$ & 2.06 & 2.02 & 2.05 & 2.88 & 2.32 & 2.63 & 4 & 1.6 \\
\hline 2gtq & $\begin{array}{l}\text { Neisseria } \\
\text { meningitidis }\end{array}$ & water & 2.09 & 2.08 & 2.00 & 2.98 & 2.26 & & 4 & 2.1 \\
\hline $2 \mathrm{hpo}$ & $\begin{array}{l}\text { Escherichia } \\
\text { coli }\end{array}$ & water & 2.06 & 2.02 & 1.97 & 2.75 & 2.08 & & 5 & 1.7 \\
\hline $2 \mathrm{hpt}$ & $\begin{array}{l}\text { Escherichia } \\
\text { coli }\end{array}$ & $\begin{array}{l}\text { 2-(3-amino-2-hydroxy-4- } \\
\text { phenyl-butyrylamino)-4- } \\
\text { methyl- pentanoic acid }\end{array}$ & 2.06 & 2.09 & 2.07 & 2.96 & 2.32 & 2.47 & 5 & 2.3 \\
\hline $2 z x g$ & $\begin{array}{l}\text { Escherichia } \\
\text { coli }\end{array}$ & $\begin{array}{l}\mathrm{n}-\{(2 \mathrm{~s})-3-[(1 r)-1- \\
\text { aminoethyl](hydroxy)pho }\end{array}$ & 2.04 & 2.05 & 1.97 & 2.89 & 2.01 & & 4 & 1.6 \\
\hline
\end{tabular}




\begin{tabular}{|c|c|c|c|c|c|c|c|c|c|c|}
\hline & & $\begin{array}{l}\text { sphoryl-2- } \\
\text { benzylpropanoyl\}-I- } \\
\text { phenylalanine }\end{array}$ & & & & & & & & \\
\hline $3 b 2 p$ & $\begin{array}{l}\text { Escherichia } \\
\text { coli }\end{array}$ & Arg & 2.05 & 2.09 & 2.03 & 2.93 & 1.81 & & 4 & 2 \\
\hline $3 b 2 x$ & $\begin{array}{l}\text { Escherichia } \\
\text { coli }\end{array}$ & Lys & 2.06 & 2.03 & 1.97 & 2.85 & 1.97 & & 4 & 1.5 \\
\hline $3 b 34$ & $\begin{array}{l}\text { Escherichia } \\
\text { coli }\end{array}$ & Phe & 2.01 & 2 & 1.90 & 2.86 & 2.06 & & 4 & 1.3 \\
\hline $3 b 37$ & $\begin{array}{l}\text { Escherichia } \\
\text { coli }\end{array}$ & Tyr & 2.04 & 2.04 & 1.95 & 2.82 & 2.26 & & 4 & 1.7 \\
\hline $3 b 3 b$ & $\begin{array}{l}\text { Escherichia } \\
\text { coli }\end{array}$ & Trp & 2 & 2.03 & 1.95 & 2.83 & 2.1 & & 4 & 1.9 \\
\hline 3ked & $\begin{array}{l}\text { Escherichia } \\
\text { coli }\end{array}$ & 2,4-diaminobutyric acid & 1.97 & 2.06 & 1.98 & 2.86 & 1.8 & & 4 & 2.3 \\
\hline 3puu & $\begin{array}{l}\text { Escherichia } \\
\text { coli }\end{array}$ & water & 2.02 & 2.11 & 2.02 & 2.81 & 2.58 & 2.61 & $\begin{array}{r}3 \\
(5) \\
\end{array}$ & 2.2 \\
\hline $3 q j x$ & $\begin{array}{l}\text { Escherichia } \\
\text { coli }\end{array}$ & water & 2 & 2.02 & 1.91 & 2.81 & 1.94 & & 4 & 1.5 \\
\hline $4 f 5 c$ & Sus scrofa & water & 1.94 & 2.02 & 1.96 & 2.00 & 2 & & 5 & 3.2 \\
\hline 4fke & Sus scrofa & water & 2.07 & 2.1 & 1.91 & 2.93 & 1.91 & & 4 & 1.9 \\
\hline $4 \mathrm{fkh}$ & Sus scrofa & water & 1.97 & 2.04 & 1.94 & 2.81 & 2.11 & & 4 & 2.1 \\
\hline $4 \mathrm{fkk}$ & Sus scrofa & $\begin{array}{l}\text { 2-(3-amino-2-hydroxy-4- } \\
\text { phenyl-butyrylamino)-4- } \\
\text { methyl- pentanoic acid }\end{array}$ & 2.16 & 2.2 & 2.02 & 2.79 & 2.2 & & 4 & 2.6 \\
\hline 4 fyq & Human & acetic acid & 2.02 & 2.05 & 1.94 & 2.99 & 2.01 & 2.49 & 5 & 1.9 \\
\hline $4 f y r$ & Human & $\begin{array}{l}\text { 2-(3-amino-2-hydroxy-4- } \\
\text { phenyl-butyrylamino)-4- } \\
\text { methyl- pentanoic acid }\end{array}$ & 2.03 & 2.06 & 1.92 & 3.00 & 2.02 & 2.54 & 4 & 1.9 \\
\hline 4 fyt & Human & $\begin{array}{l}(2 \mathrm{~s}, 3 \mathrm{r})-3 \text {-amino-2- } \\
\text { hydroxy-5- } \\
\text { methylhexanoic acid }\end{array}$ & 2.03 & 2.09 & 1.94 & 3.02 & 1.95 & 2.3 & 5 & 1.9 \\
\hline 4hom & Sus scrofa & water & 2.06 & 2.08 & 1.98 & 2.91 & 2.13 & & 4 & 1.9 \\
\hline 4naq & Sus scrofa & water & 1.91 & 2.09 & 1.91 & 2.74 & 1.89 & & 4 & 2.1 \\
\hline $4 \mathrm{nz} 8$ & Sus scrofa & water & 1.96 & 2.13 & 1.96 & 2.88 & 2.15 & & 4 & 2 \\
\hline 4ou3 & Sus scrofa & water & 2.05 & 2.05 & 2.05 & 2.75 & 2.22 & & 4 & 2 \\
\hline 4pu2 & $\begin{array}{l}\text { Neisseria } \\
\text { meningitidis }\end{array}$ & leucine phosphonic acid & 1.98 & 2.04 & 2.29 & 3.04 & 2.12 & 2.27 & 5 & 2.1 \\
\hline $4 p v b$ & $\begin{array}{l}\text { Neisseria } \\
\text { meningitidis }\end{array}$ & $\begin{array}{l}\text { [(1s)-1-amino-3- } \\
\text { methylbutyl]phosphonic } \\
\text { acid }\end{array}$ & 2.06 & 2.12 & 2.06 & 2.91 & 2.06 & 2.11 & 5 & 2.1 \\
\hline 4pw4 & $\begin{array}{l}\text { Neisseria } \\
\text { meningitidis }\end{array}$ & $\begin{array}{l}\text { [(1r)-1-amino-3- } \\
\text { phenylpropyl]phosphonic } \\
\text { acid }\end{array}$ & 2.11 & 2.14 & 1.99 & 2.76 & 2.28 & 2.34 & 5 & 1.9 \\
\hline $4 q 4 e$ & $\begin{array}{l}\text { Escherichia } \\
\text { coli }\end{array}$ & actinonin & 2.06 & 2.12 & 2.02 & 2.84 & 2.05 & 2.21 & 5 & 1.9 \\
\hline $4 q 4 i$ & $\begin{array}{l}\text { Escherichia } \\
\text { coli }\end{array}$ & $\begin{array}{l}2 \mathrm{~s}, 3 \mathrm{r})-3 \text {-amino-2- } \\
\text { hydroxy-5- } \\
\text { methylhexanoic acid }\end{array}$ & 2.03 & 2.13 & 2.01 & 2.93 & 1.97 & 2.74 & 5 & 2.3 \\
\hline $4 q h p$ & $\begin{array}{l}\text { Neisseria } \\
\text { meningitidis }\end{array}$ & $\begin{array}{l}\text { 2s)-2-[4- } \\
\text { (aminomethyl)benzyl]-3- } \\
\text { [(r)-[(1r)-1-amino-3- } \\
\text { phenylpropyl](hydroxy)ph } \\
\text { osphoryl]propanoic acid }\end{array}$ & 2.08 & 2.14 & 1.92 & 2.63 & 2.21 & 2.22 & 5 & 1.6 \\
\hline 4qir & $\begin{array}{l}\text { Neisseria } \\
\text { meningitidis }\end{array}$ & $\begin{array}{l}\text { 3-\{[(r)-1-amino-3- } \\
\text { (pyridin-3- } \\
\text { yl)propyl](hydroxy) } \\
\text { phosphoryl\}-(s)-2- } \\
\text { benzylpropanoic acid }\end{array}$ & 2.04 & 2.07 & 1.92 & 2.56 & 2.18 & 2.37 & 5 & 1.7 \\
\hline 4qme & $\begin{array}{l}\text { Neisseria } \\
\text { meningitidis }\end{array}$ & $\begin{array}{l}\text { (2s)-3-[(s)-[(1r)-1-amino- } \\
\text { 3-phenylpropyl](hydroxy) }\end{array}$ & 2.05 & 2.07 & 1.94 & 2.64 & 2.16 & 2.35 & 5 & 1.6 \\
\hline
\end{tabular}




\begin{tabular}{|c|c|c|c|c|c|c|c|c|c|c|}
\hline & & $\begin{array}{l}\text { phosphoryl]-2- } \\
\text { benzylpropanoic acid }\end{array}$ & & & & & & & & \\
\hline 4qpe & $\begin{array}{l}\text { Neisseria } \\
\text { meningitidis }\end{array}$ & $\begin{array}{l}\text { [(1r)-1-amino-2- } \\
\text { (cyclohexylamino)ethyl]p } \\
\text { hosphonic acid }\end{array}$ & 2.09 & 2.09 & 2.09 & 2.77 & 2.27 & 2.35 & 5 & 2 \\
\hline 4quo & $\begin{array}{l}\text { Neisseria } \\
\text { meningitidis }\end{array}$ & $\begin{array}{l}\text { 2s)-2-[3- } \\
\text { (aminomethyl)benzyl]-3- } \\
\text { [(r)-[(1r)-1-amino-3- } \\
\text { phenylpropyl](hydroxy)ph } \\
\text { osphoryl]propanoic acid }\end{array}$ & 2.12 & 2.13 & 1.97 & 2.61 & 2.17 & 2.19 & 5 & 1.7 \\
\hline $4 \mathrm{xmt}$ & $\begin{array}{l}\text { Escherichia } \\
\text { coli }\end{array}$ & iaminopropanoic acid & 2.08 & 2.16 & 2.03 & 2.75 & 2.1 & 2.31 & 5 & 2 \\
\hline $4 \times m u$ & $\begin{array}{l}\text { Escherichia } \\
\text { coli }\end{array}$ & Ala & 2.16 & 2.05 & 2.02 & 2.83 & 2.39 & & 4 & 2.9 \\
\hline $4 \times \mathrm{xmv}$ & $\begin{array}{l}\text { Escherichia } \\
\text { coli }\end{array}$ & Arg & 2.06 & 2.07 & 2.04 & 2.80 & 1.81 & & 4 & 2.9 \\
\hline $4 \times m w$ & $\begin{array}{l}\text { Escherichia } \\
\text { coli }\end{array}$ & Asp & 2.05 & 2.12 & 2.01 & 2.81 & 2.55 & 1.76 & 5 & 2.2 \\
\hline $4 \times m x$ & $\begin{array}{l}\text { Escherichia } \\
\text { coli }\end{array}$ & $\begin{array}{l}\text { 2-(3-amino-2-hydroxy-4- } \\
\text { phenyl-butyrylamino)-4- } \\
\text { methyl- pentanoic acid }\end{array}$ & 2.02 & 2.12 & 2.03 & 2.76 & 1.96 & 2.52 & 5 & 2.3 \\
\hline $4 \times \mathrm{xmz}$ & $\begin{array}{l}\text { Escherichia } \\
\text { coli }\end{array}$ & $\begin{array}{l}\text { 2-(3-amino-2-hydroxy-4- } \\
\text { phenyl-butyrylamino)-4- } \\
\text { methyl- pentanoic acid }\end{array}$ & 2.06 & 2.09 & 2.01 & 2.80 & 1.77 & & 4 & 2.2 \\
\hline $4 \times n 1$ & $\begin{array}{l}\text { Escherichia } \\
\text { coli }\end{array}$ & 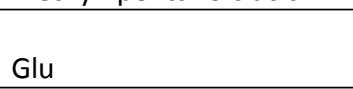 & 2.07 & 2.16 & 2.01 & 2.83 & 2.27 & 2.08 & 5 & 2.2 \\
\hline $4 \times n 2$ & $\begin{array}{l}\text { Escherichia } \\
\text { coli }\end{array}$ & Leu & 2.02 & 2.08 & 1.97 & 2.83 & 1.82 & & 4 & 2.1 \\
\hline $4 \times n 4$ & $\begin{array}{l}\text { Escherichia } \\
\text { coli }\end{array}$ & Met & 1.99 & 2.08 & 1.97 & 2.91 & 1.81 & & 4 & 2 \\
\hline $4 \times n 5$ & $\begin{array}{l}\text { Escherichia } \\
\text { coli }\end{array}$ & Phe & 2.14 & 2.16 & 2.06 & 2.73 & 1.82 & & 4 & 2.7 \\
\hline $4 \times n 7$ & $\begin{array}{l}\text { Escherichia } \\
\text { coli }\end{array}$ & laminopropanoic acid & 2.18 & 2.36 & 2.07 & 2.85 & 2.28 & & 4 & 2.2 \\
\hline $4 \times n 8$ & $\begin{array}{l}\text { Escherichia } \\
\text { coli }\end{array}$ & Ala & 2 & 2.05 & 1.93 & 2.87 & 1.94 & & 4 & 1.9 \\
\hline $4 \times n 9$ & $\begin{array}{l}\text { Escherichia } \\
\text { coli }\end{array}$ & beta Ala & 2.1 & 2.08 & 2.17 & 2.86 & 1.84 & & 4 & 2.8 \\
\hline 4xna & $\begin{array}{l}\text { Escherichia } \\
\text { coli }\end{array}$ & $\begin{array}{l}\text { (3s)-3,7- } \\
\text { diaminoheptanoic acid }\end{array}$ & 2.07 & 2.16 & 1.92 & 2.76 & 1.99 & 2.25 & 5 & 2.4 \\
\hline $4 \times n b$ & $\begin{array}{l}\text { Escherichia } \\
\text { coli }\end{array}$ & $\begin{array}{l}\text { (3s)-3-amino-4- } \\
\text { phenylbutanoic acid }\end{array}$ & 2.06 & 2.07 & 1.98 & 2.84 & 2 & 2.63 & 4 & 2 \\
\hline 4xnd & $\begin{array}{l}\text { Escherichia } \\
\text { coli }\end{array}$ & $\begin{array}{l}\text { (3s)-3-amino-4-(1h-indol- } \\
\text { 3-yl)butanoic acid }\end{array}$ & 2.02 & 2.07 & 1.93 & 2.82 & 1.88 & & 4 & 1.9 \\
\hline $4 \times 03$ & $\begin{array}{l}\text { Escherichia } \\
\text { coli }\end{array}$ & Leu & 1.98 & 2.02 & 1.94 & 2.92 & 2.01 & & 5 & 2 \\
\hline $4 \times 04$ & $\begin{array}{l}\text { Escherichia } \\
\text { coli }\end{array}$ & Met & 2.06 & 2.12 & 2.08 & 2.74 & 1.76 & & 4 & 2.2 \\
\hline $4 \times 05$ & $\begin{array}{l}\text { Escherichia } \\
\text { coli }\end{array}$ & Glu & 2.03 & 2.05 & 2.01 & 2.84 & 1.85 & & 4 & 2 \\
\hline $5 \mathrm{dll}$ & $\begin{array}{l}\text { Francisella } \\
\text { tularensis } \\
\text { subsp. } \\
\text { tularensis }\end{array}$ & & 2.16 & 2.15 & 2.00 & 2.00 & & & 4 & 2.5 \\
\hline 5 dyf & $\begin{array}{l}\text { Neisseria } \\
\text { meningitidis }\end{array}$ & $\begin{array}{l}\text { [(1r)-1-amino-2- } \\
\text { (benzylamino)ethyl]phos } \\
\text { phonic acid }\end{array}$ & 2.11 & 2.04 & 2.09 & 2.80 & 2.13 & 2.28 & 5 & 1.9 \\
\hline $5 \lg 6$ & Sus scrofa & water & 2.1 & 2.17 & 2.14 & 2.96 & 2.37 & & 4 & 2.5 \\
\hline $5 \mathrm{mfr}$ & $\begin{array}{l}\text { Escherichia } \\
\text { coli }\end{array}$ & $\begin{array}{l}{[(7 \sim\{s\})-6,6-\text { bis(oxidanyl)- }} \\
5,7,8,9- \\
\text { tetrahydrobenzo[7]annul }\end{array}$ & 2.04 & 2.03 & 1.96 & 2.86 & 1.94 & 1.86 & 5 & 1.4 \\
\hline
\end{tabular}




\begin{tabular}{|c|c|c|c|c|c|c|c|c|c|c|}
\hline & & $\begin{array}{l}\text { en-7-yl]azanium and } \\
\text { glycerol }\end{array}$ & & & & & & & & \\
\hline $5 \mathrm{mfs}$ & $\begin{array}{l}\text { Escherichia } \\
\text { coli }\end{array}$ & $\begin{array}{l}{[(7 \sim\{s\})-6,6-b i s(\text { oxidanyl)- }} \\
\text { 4-phenyl-5,7,8,9- } \\
\text { tetrahydrobenzo[7]annul } \\
\text { en-7-yl]azanium }\end{array}$ & 2.03 & 2.04 & 2.00 & 2.94 & 1.95 & & 4 & 1.6 \\
\hline $5 \mathrm{mft}$ & $\begin{array}{l}\text { Escherichia } \\
\text { coli }\end{array}$ & $\begin{array}{l}{[(7 \sim\{s\})-1-\text { bromanyl-6,6- }} \\
\text { bis(oxidanyl)-4-phenyl- } \\
5,7,8,9- \\
\text { tetrahydrobenzo[7]annul } \\
\text { en-7-yl]azanium }\end{array}$ & 2.04 & 2.05 & 1.96 & 2.88 & 1.96 & & 4 & 1.6 \\
\hline 5 yo1 & $\begin{array}{l}\text { Escherichia } \\
\text { coli }\end{array}$ & 1 & 2.16 & 2.18 & 2.06 & 2.69 & 2.19 & 2.6 & 4 & 2.5 \\
\hline $5 \mathrm{yq} 1$ & $\begin{array}{l}\text { Escherichia } \\
\text { coli }\end{array}$ & o-methyl-I-tyrosine & 2.03 & 2.04 & 1.98 & 2.84 & 1.91 & & 4 & 1.6 \\
\hline $5 y q 2$ & $\begin{array}{l}\text { Escherichia } \\
\text { coli }\end{array}$ & water & 2.04 & 2.06 & 1.94 & 2.75 & 1.99 & & 4 & 1.6 \\
\hline $5 y q b$ & $\begin{array}{l}\text { Escherichia } \\
\text { coli }\end{array}$ & o-methyl-I-tyrosine & 2.02 & 2.04 & 1.94 & 2.86 & 1.88 & & 4 & 1.6 \\
\hline $5 z i 5$ & $\begin{array}{l}\text { Legionella } \\
\text { pneumophil }\end{array}$ & water & 2.17 & 2.26 & 2.03 & 2.73 & 2.1 & & 4 & 2.6 \\
\hline 6atk & Human & & 2.2 & 2.23 & 1.97 & 2.82 & & & 3 & 3.5 \\
\hline 6buy & Sus scrofa & water & 2.04 & 2.15 & 1.92 & 2.87 & 2.05 & & 4 & 2.1 \\
\hline $6 b v 0$ & Sus scrofa & water & 2.11 & 2.09 & 2.01 & 2.85 & 2.17 & & 4 & 1.9 \\
\hline $6 \mathrm{bv} 1$ & Sus scrofa & water & 2.13 & 2.13 & 1.92 & 2.81 & 2.19 & & 4 & 2 \\
\hline $6 \mathrm{bv} 2$ & Sus scrofa & water & 1.96 & 1.98 & 1.80 & 3.01 & 2.15 & & 4 & 2.1 \\
\hline $6 \mathrm{bv} 3$ & Sus scrofa & & 2.06 & 1.94 & 1.84 & 2.97 & & & 4 & 2.2 \\
\hline $6 \mathrm{bv} 4$ & Sus scrofa & water & 2.11 & 2.02 & 1.85 & 2.94 & 2.24 & & 4 & 2 \\
\hline $6 g 8 b$ & $\begin{array}{l}\text { Escherichia } \\
\text { coli }\end{array}$ & $\begin{array}{l}\text { [(7 \{s\})-1-bromanyl-6,6- } \\
\text { bis(oxidanyl)-4-phenyl- } \\
5,7,8,9- \\
\text { tetrahydrobenzo[7]annul } \\
\text { en-7-yl]azanium }\end{array}$ & 2.06 & 2.14 & 2.00 & 2.91 & 1.95 & & 4 & 2.4 \\
\hline
\end{tabular}

\section{Thermolysin}

\begin{tabular}{|c|c|c|c|c|c|c|c|c|c|c|}
\hline PDB & organism & ligand & $\mathrm{Zn}-\mathrm{H}$ & $\mathrm{Zn}-\mathrm{H}$ & $\begin{array}{l}\mathrm{Zn}-\mathrm{E}(0) \\
\text { closer }\end{array}$ & $\begin{array}{l}\mathrm{Zn-} \\
\mathrm{E}(0)\end{array}$ & $\begin{array}{l}\text { Zn- } \\
\text { ligand } \\
\text { closest }\end{array}$ & $\begin{array}{l}\mathrm{Zn-} \\
\text { ligand }\end{array}$ & $\mathrm{CN}$ & $\begin{array}{l}\text { Resolu- } \\
\text { tion/Å }\end{array}$ \\
\hline $1 \mathrm{esp}$ & Bacillus cereus & water & 2.08 & 2.01 & 1.99 & 2.95 & 2.19 & & 4 & 2.8 \\
\hline $1 \mathrm{fj} 3$ & $\begin{array}{l}\text { Bacillus } \\
\text { thermoproteolyticus }\end{array}$ & water & 2 & 2.01 & 2.22 & 2.29 & 2.52 & & 5 & 2 \\
\hline $1 \mathrm{fjo}$ & $\begin{array}{l}\text { Bacillus } \\
\text { thermoproteolyticus }\end{array}$ & water & 2 & 2.01 & 2.23 & 2.28 & 1.96 & & 5 & 2 \\
\hline $1 \mathrm{fjq}$ & $\begin{array}{l}\text { Bacillus } \\
\text { thermoproteolyticus }\end{array}$ & water & 2.24 & 2.29 & 2.56 & 2.79 & 2.27 & & 4 & 1.7 \\
\hline $1 \mathrm{fjt}$ & Bacillus cereus & water & 2.02 & 2.03 & 2.25 & 2.26 & 1.95 & & 5 & 2.2 \\
\hline $1 \mathrm{fju}$ & $\begin{array}{l}\text { Bacillus } \\
\text { thermoproteolyticus }\end{array}$ & water & 2.09 & 2.08 & 2.32 & 2.34 & 2.12 & & 5 & 2 \\
\hline $1 \mathrm{fjv}$ & $\begin{array}{l}\text { Bacillus } \\
\text { thermoproteolyticus }\end{array}$ & water & 2.02 & 2.03 & 2.24 & 2.27 & 1.96 & & 5 & 2 \\
\hline $1 \mathrm{fjw}$ & $\begin{array}{l}\text { Bacillus } \\
\text { thermoproteolyticus }\end{array}$ & water & 1.99 & 2 & 2.25 & 2.26 & 2.51 & & 5 & 1.9 \\
\hline 1gxw & $\begin{array}{l}\text { Bacillus } \\
\text { thermoproteolyticus }\end{array}$ & & 2.19 & 2.09 & 2.17 & 3.17 & & & 3 & 2.18 \\
\hline $1 \mathrm{kei}$ & $\begin{array}{l}\text { Bacillus } \\
\text { thermoproteolyticus }\end{array}$ & & 2.05 & 2 & 1.84 & 2.97 & & & 3 & 1.6 \\
\hline
\end{tabular}




\begin{tabular}{|c|c|c|c|c|c|c|c|c|c|c|}
\hline $1 \mathrm{kr} 6$ & $\begin{array}{l}\text { Bacillus } \\
\text { thermoproteolyticus }\end{array}$ & D-Glu & 2.06 & 2.03 & 1.92 & 3.03 & 2.15 & 2.4 & 5 & 1.8 \\
\hline $1 \mathrm{kro}$ & $\begin{array}{l}\text { Bacillus } \\
\text { thermoproteolyticus }\end{array}$ & D-Thr & 2.07 & 2.02 & 1.93 & 3.02 & 2.05 & 2.51 & $4(5)$ & 1.7 \\
\hline $1 \mathrm{ks} 7$ & $\begin{array}{l}\text { Bacillus } \\
\text { thermoproteolyticus }\end{array}$ & D-Asp & 2.07 & 2.02 & 1.88 & 3.01 & 2.3 & 2.47 & 5 & 1.7 \\
\hline 1 kto & $\begin{array}{l}\text { Bacillus } \\
\text { thermoproteolyticus }\end{array}$ & D-Ala & 2.03 & 1.98 & 1.95 & 2.93 & 2 & & 4 & 1.9 \\
\hline $1 \operatorname{lnf}$ & $\begin{array}{l}\text { Bacillus } \\
\text { thermoproteolyticus }\end{array}$ & water & 1.98 & 1.99 & 2.24 & 2.38 & 2.28 & & 4 & 1.7 \\
\hline $1 \mathrm{npc}$ & Bacillus cereus & water & 2.05 & 2 & 2.10 & 2.48 & 1.72 & & 5 & 2 \\
\hline 10 os & $\begin{array}{l}\text { Bacillus } \\
\text { thermoproteolyticus }\end{array}$ & $\begin{array}{l}\text { n-\{(2r)-3-[(s)- } \\
{[(1 r)-1-a m i n o-2-} \\
\text { phenylethyl](hyd } \\
\text { roxy) } \\
\text { phosphoryl]-2- } \\
\text { benzylpropanoyl\} } \\
\text {-I-phenylalanine }\end{array}$ & 2.03 & 2.02 & 1.94 & 2.95 & 1.9 & & 4 & 2.1 \\
\hline $1 p e 5$ & $\begin{array}{l}\text { Bacillus } \\
\text { thermoproteolyticus }\end{array}$ & $\begin{array}{l}\text { (6-methyl-3,4- } \\
\text { dihydro-2h- } \\
\text { chromen-2- } \\
\text { yl)methylphosphi } \\
\text { nate }\end{array}$ & 2 & 2.05 & 2.14 & 2.53 & 1.89 & & 5 & 1.7 \\
\hline 1pe7 & $\begin{array}{l}\text { Bacillus } \\
\text { thermoproteolyticus }\end{array}$ & $\begin{array}{l}\text { 2-(4- } \\
\text { methylphenoxy)e } \\
\text { thylphosphinate }\end{array}$ & 2.04 & 2.02 & 2.15 & 2.61 & 1.99 & & 4 & 1.82 \\
\hline $1 \mathrm{pe} 8$ & $\begin{array}{l}\text { Bacillus } \\
\text { thermoproteolyticus }\end{array}$ & $\begin{array}{l}\text { 2- } \\
\text { ethoxyethylphos } \\
\text { phinate }\end{array}$ & 1.97 & 1.96 & 1.98 & 2.76 & 2.02 & & 4 & 1.8 \\
\hline $1 \mathrm{qfO}$ & $\begin{array}{l}\text { Bacillus } \\
\text { thermoproteolyticus }\end{array}$ & $\begin{array}{l}\text { (2-sulfanyl-3- } \\
\text { phenylpropanoyl } \\
\text { )-phe-tyr }\end{array}$ & 2.07 & 2.22 & 1.96 & 3.14 & 2.4 & 2.38 & 5 & 2.2 \\
\hline $1 q f 1$ & $\begin{array}{l}\text { Bacillus } \\
\text { thermoproteolyticus }\end{array}$ & $\begin{array}{l}\text { [2(r,s)-2- } \\
\text { sulfanylheptanoy } \\
\text { I]-phe-ala }\end{array}$ & 2.06 & 2.19 & 1.96 & 3.18 & 2.29 & 2.41 & 5 & 2 \\
\hline $1 q f 2$ & $\begin{array}{l}\text { Bacillus } \\
\text { thermoproteolyticus }\end{array}$ & $\begin{array}{l}\text { [(2s)-2-sulfanyl- } \\
\text { 3- } \\
\text { phenylpropanoyl } \\
\text { ]-gly-(5- } \\
\text { phenylproline) }\end{array}$ & 2.03 & 2.11 & 1.94 & 2.99 & 2.29 & & 4 & 2.06 \\
\hline 1 thl & $\begin{array}{l}\text { Bacillus } \\
\text { thermoproteolyticus }\end{array}$ & $\begin{array}{l}\text { n-(\{1-[(2s)-2- } \\
\text { carboxy-4- } \\
\text { phenylbutyl]cycl } \\
\text { opentyl\}carbonyl } \\
\text { )-I-tryptophan }\end{array}$ & 2.02 & 2.04 & 1.99 & 2.97 & 2.01 & & 4 & 1.7 \\
\hline $1 \mathrm{tli}$ & $\begin{array}{l}\text { Bacillus } \\
\text { thermoproteolyticus }\end{array}$ & water & 2.03 & 2 & 2.21 & 2.28 & 2.09 & & 5 & 2.05 \\
\hline 1tlp & $\begin{array}{l}\text { Bacillus } \\
\text { thermoproteolyticus }\end{array}$ & $\begin{array}{l}\text { n-alpha-I- } \\
\text { rhamnopyranosy } \\
\text { loxy(hydroxypho } \\
\text { sphinyl)-I- leucyl- } \\
\text { I-tryptophan }\end{array}$ & 2.19 & 2.22 & 1.95 & 2.68 & 1.75 & & 5 & 2.3 \\
\hline $1 \mathrm{tlx}$ & $\begin{array}{l}\text { Bacillus } \\
\text { thermoproteolyticus }\end{array}$ & water & 1.99 & 2.02 & 2.24 & 2.27 & 2.05 & & 5 & 2.1 \\
\hline $1 \mathrm{tmn}$ & $\begin{array}{l}\text { Bacillus } \\
\text { thermoproteolyticus }\end{array}$ & & 1.95 & 2.04 & 1.95 & 2.87 & 2.01 & 2.41 & 5 & 1.9 \\
\hline $1 \mathrm{u} 4 \mathrm{~g}$ & $\begin{array}{l}\text { Pseudomonas } \\
\text { aeruginosa }\end{array}$ & $\begin{array}{l}\text { n-(1-carboxy-3- } \\
\text { phenylpropyl)ph } \\
\text { enylalanyl-alpha- } \\
\text { asparagine }\end{array}$ & 2.07 & 2.05 & 2.08 & 2.89 & 2.71 & 1.9 & 5 & 1.4 \\
\hline $1 y 3 g$ & $\begin{array}{l}\text { Bacillus } \\
\text { thermoproteolyticus }\end{array}$ & $\begin{array}{l}\text { (2s)-2- } \\
\{[(\text { aminomethyl)( }\end{array}$ & 1.98 & 2.03 & 2.07 & 2.88 & 1.93 & & 4 & 2.1 \\
\hline
\end{tabular}




\begin{tabular}{|c|c|c|c|c|c|c|c|c|c|c|}
\hline & & $\begin{array}{l}\text { dihydroxy)silyl]m } \\
\text { ethyl\}-4- } \\
\text { methylpentanal }\end{array}$ & & & & & & & & \\
\hline $1 z 9 g$ & $\begin{array}{l}\text { Bacillus } \\
\text { thermoproteolyticus }\end{array}$ & $\begin{array}{l}\text { (r)-retro- } \\
\text { thiorphan }\end{array}$ & 2.06 & 2.02 & 2.17 & 2.81 & 2.32 & & 4 & 1.7 \\
\hline $1 z d p$ & $\begin{array}{l}\text { Bacillus } \\
\text { thermoproteolyticus }\end{array}$ & $\begin{array}{l}\text { (2- } \\
\text { mercaptomethyl- } \\
\text { 3-phenyl- } \\
\text { propionyl)- } \\
\text { glycine }\end{array}$ & 2.14 & 2.1 & 2.00 & 2.95 & 2.41 & & 4 & 1.7 \\
\hline $2 a 7 g$ & $\begin{array}{l}\text { Bacillus } \\
\text { thermoproteolyticus }\end{array}$ & acetic acid & 2.05 & 2.04 & 2.00 & 2.99 & 2.25 & 2.33 & 5 & 1.85 \\
\hline $2 g 4 z$ & $\begin{array}{l}\text { Bacillus } \\
\text { thermoproteolyticus }\end{array}$ & chloride ion & 1.99 & 2.11 & 1.97 & 2.84 & 2.41 & & 4 & 1.98 \\
\hline 2036 & Human & water & 2.16 & 2.17 & 2.03 & 2.98 & 2.07 & & 4 & 1.95 \\
\hline $203 e$ & Rattus norvegicus & water & 2.15 & 2.21 & 2.16 & 2.68 & 2.34 & & $4(5)$ & 2.2 \\
\hline $2 \mathrm{tli}$ & $\begin{array}{l}\text { Bacillus } \\
\text { thermoproteolyticus }\end{array}$ & water & 2.03 & 2.03 & 2.23 & 2.29 & 2.08 & & 5 & 1.95 \\
\hline $2 \mathrm{tlx}$ & $\begin{array}{l}\text { Bacillus } \\
\text { thermoproteolyticus }\end{array}$ & water & 2.18 & 2.22 & 2.65 & 2.68 & 2.65 & 2.68 & $3(6)$ & 1.65 \\
\hline $2 \mathrm{tmn}$ & $\begin{array}{l}\text { Bacillus } \\
\text { thermoproteolyticus }\end{array}$ & $\begin{array}{l}\mathrm{n}^{\sim} 2^{\sim-} \\
\text { phosphono-I- } \\
\text { leucinamide }\end{array}$ & 2.1 & 2.12 & 2.15 & 2.89 & 2.07 & & 4 & 1.6 \\
\hline $2 v q x$ & $\begin{array}{l}\text { Serratia } \\
\text { proteamaculans }\end{array}$ & water & 2.21 & 2.11 & 2.12 & 3.18 & 2.09 & 2.17 & 5 & 1.82 \\
\hline $2 w h z$ & $\begin{array}{l}\text { Bacillus } \\
\text { thermoproteolyticus }\end{array}$ & water & 2 & 2.09 & 2.03 & 2.99 & 2.27 & & 5 & 1.75 \\
\hline $2 \mathrm{wiO}$ & $\begin{array}{l}\text { Bacillus } \\
\text { thermoproteolyticus }\end{array}$ & water & 2.08 & 2.07 & 1.96 & 3.01 & 2.24 & & 4 & 1.95 \\
\hline $3 b 2 p$ & Escherichia coli & Lys & 2.05 & 2.09 & 2.03 & 2.93 & 1.81 & & 4 & 2 \\
\hline $3 b 2 x$ & Escherichia coli & Lys & 2.06 & 2.03 & 1.97 & 2.85 & 1.97 & & 4 & 1.5 \\
\hline $3 b 34$ & Escherichia coli & Phe & 2.01 & 2 & 1.90 & 2.86 & 2.06 & & 4 & 1.3 \\
\hline $3 b 37$ & Escherichia coli & Tyr & 2.04 & 2.04 & 1.95 & 2.82 & 2.26 & & 4 & 1.7 \\
\hline $3 b 3 b$ & Escherichia coli & Trp & 2 & 2.03 & 1.95 & 2.83 & 2.1 & & 4 & 1.85 \\
\hline $3 \mathrm{dbk}$ & $\begin{array}{l}\text { Pseudomonas } \\
\text { aeruginosa }\end{array}$ & $\begin{array}{l}\text { n-alpha-l- } \\
\text { rhamnopyranosy } \\
\text { loxy(hydroxypho } \\
\text { sphinyl)-I- leucyl- } \\
\text { I-tryptophan }\end{array}$ & 2.05 & 2.02 & 1.99 & 2.98 & 1.91 & & 4 & 1.4 \\
\hline $3 d n z$ & $\begin{array}{l}\text { Bacillus } \\
\text { thermoproteolyticus }\end{array}$ & & 2.03 & 2.05 & 2.12 & 2.94 & & & 3 & 1.2 \\
\hline $3 \mathrm{do} 0$ & $\begin{array}{l}\text { Bacillus } \\
\text { thermoproteolyticus }\end{array}$ & & 2.05 & 2.08 & 2.01 & 2.95 & & & 3 & 1.36 \\
\hline 3do1 & $\begin{array}{l}\text { Bacillus } \\
\text { thermoproteolyticus }\end{array}$ & & 2.02 & 2.06 & 1.93 & 3.03 & & & 3 & 1.33 \\
\hline 3 do2 & $\begin{array}{l}\text { Bacillus } \\
\text { thermoproteolyticus }\end{array}$ & & 2.03 & 2.07 & 2.07 & 2.95 & & & 3 & 1.22 \\
\hline $3 f 28$ & $\begin{array}{l}\text { Bacillus } \\
\text { thermoproteolyticus }\end{array}$ & $\begin{array}{l}\text { 2- } \\
\text { [(cyclopropylcarb } \\
\text { onyl)oxy]-3- } \\
\text { methylbenzoic } \\
\text { acid }\end{array}$ & 2.02 & 2.03 & 2.04 & 3.00 & 1.99 & 2.66 & 5 & 1.68 \\
\hline $3 f 2 p$ & $\begin{array}{l}\text { Bacillus } \\
\text { thermoproteolyticus }\end{array}$ & $\begin{array}{l}\text { 3-methyl-2- } \\
\text { (propanoyloxy)b } \\
\text { enzoic acid }\end{array}$ & 1.98 & 2.09 & 2.09 & 2.92 & 2.06 & 2.59 & $4(5)$ & 1.95 \\
\hline $3 f c q$ & $\begin{array}{l}\text { Bacillus } \\
\text { thermoproteolyticus }\end{array}$ & $\begin{array}{l}\text { 2-(acetyloxy)-3- } \\
\text { methylbenzoic } \\
\text { acid }\end{array}$ & 1.95 & 2.02 & 1.97 & 3.05 & 2.17 & 2.7 & $4(5)$ & 1.75 \\
\hline $3 \mathrm{fgd}$ & $\begin{array}{l}\text { Bacillus } \\
\text { thermoproteolyticus }\end{array}$ & $\begin{array}{l}\mathrm{n}- \\
\text { (phenylcarbonyl) } \\
\text {-beta-alanine }\end{array}$ & 1.99 & 2 & 1.98 & 3.02 & 1.97 & 2.63 & $4(5)$ & 1.33 \\
\hline
\end{tabular}




\begin{tabular}{|c|c|c|c|c|c|c|c|c|c|c|}
\hline $3 f \mid f$ & $\begin{array}{l}\text { Bacillus } \\
\text { thermoproteolyticus }\end{array}$ & $\begin{array}{l}\mathrm{n}-[(\mathrm{s})- \\
(\{[(\text { benzyloxy)car } \\
\text { bonyl]amino\} } \\
\text { methyl)(hydroxy) } \\
\text { phosphoryl]-I- } \\
\text { valyl-I-leucine }\end{array}$ & 2.08 & 2.06 & 1.94 & 2.82 & 1.97 & & 4 & 1.97 \\
\hline 3 for & $\begin{array}{l}\text { Bacillus } \\
\text { thermoproteolyticus }\end{array}$ & $\begin{array}{l}\text { (2r)-2-benzyl-3- } \\
\text { nitropropanamid } \\
\text { e }\end{array}$ & 2.12 & 2.11 & 2.10 & 2.95 & 2.25 & 2.63 & $4(5)$ & 1.93 \\
\hline $3 f v 4$ & $\begin{array}{l}\text { Bacillus } \\
\text { thermoproteolyticus }\end{array}$ & $\begin{array}{l}\text { n-[(s)- } \\
\text { (\{[(benzyloxy)car } \\
\text { bonyl]amino } \\
\text { \}methyl)(hydroxy } \\
\text { )phosphoryl]-I- } \\
\text { phenylalanyl--- } \\
\text { leucine }\end{array}$ & 2.06 & 2.03 & 1.94 & 2.97 & 1.99 & & 4 & 1.56 \\
\hline $3 \mathrm{fvp}$ & $\begin{array}{l}\text { Bacillus } \\
\text { thermoproteolyticus }\end{array}$ & $\begin{array}{l}\mathrm{n}-[(\mathrm{s})- \\
\text { (\{[(benzyloxy)car } \\
\text { bonyl]amino } \\
\text { \}methyl)(hydroxy } \\
\text { )phosphoryl]-I- } \\
\text { alanyl-I-leucine }\end{array}$ & 1.98 & 2.02 & 1.97 & 2.97 & 1.98 & & 4 & 1.41 \\
\hline $3 f x p$ & $\begin{array}{l}\text { Bacillus } \\
\text { thermoproteolyticus }\end{array}$ & $\begin{array}{l}\mathrm{n}^{\sim} 2^{\sim}-[(2 \mathrm{~s})-2-\{[1- \\
(4- \\
\text { carboxybenzyl)- } \\
1 \mathrm{~h}-1,2,3-\text { triazol- } \\
4-\mathrm{y}] \mathrm{lmethyl \} -3-} \\
\text { methylbutanoyl]- } \\
\text { l-lysine }\end{array}$ & 1.92 & 1.98 & 1.91 & 2.94 & 1.93 & & 4 & 2.05 \\
\hline 3157 & $\begin{array}{l}\text { Bacillus } \\
\text { thermoproteolyticus }\end{array}$ & water & 1.91 & 2.03 & 2.10 & 2.88 & 2.43 & & 4 & 1.98 \\
\hline $3 \mathrm{~ms} 3$ & $\begin{array}{l}\text { Bacillus } \\
\text { thermoproteolyticus }\end{array}$ & water & 1.95 & 2.02 & 1.99 & 3.03 & 2.16 & & 4 & 1.54 \\
\hline $3 \mathrm{msa}$ & $\begin{array}{l}\text { Bacillus } \\
\text { thermoproteolyticus }\end{array}$ & water & 2 & 2.03 & 1.90 & 3.13 & 1.87 & & 5 & 1.66 \\
\hline $3 \mathrm{msf}$ & $\begin{array}{l}\text { Bacillus } \\
\text { thermoproteolyticus }\end{array}$ & water & 2.06 & 1.97 & 1.90 & 3.00 & 2.35 & & 4 & 2.09 \\
\hline $3 \mathrm{msn}$ & $\begin{array}{l}\text { Bacillus } \\
\text { thermoproteolyticus }\end{array}$ & water & 2.02 & 2.14 & 1.95 & 2.97 & 2.3 & 2.63 & $4(5)$ & 1.97 \\
\hline $3 n 21$ & $\begin{array}{l}\text { Bacillus } \\
\text { thermoproteolyticus }\end{array}$ & water & 2.04 & 2.09 & 2.00 & 3.01 & 1.97 & 2.39 & 5 & 1.87 \\
\hline $3 \mathrm{nn} 7$ & $\begin{array}{l}\text { Bacillus } \\
\text { thermoproteolyticus }\end{array}$ & water & 1.87 & 2.04 & 1.96 & 3.06 & 2.24 & 2.63 & $4(5)$ & 2.05 \\
\hline $3 n q x$ & $\begin{array}{l}\text { Pseudoalteromonas } \\
\text { sp. }\end{array}$ & & 2.05 & 2.04 & 2.02 & 3.00 & & & 3 & 1.7 \\
\hline $3 p 7 p$ & $\begin{array}{l}\text { Bacillus } \\
\text { thermoproteolyticus }\end{array}$ & water & 2.01 & 2.01 & 1.96 & 2.99 & 1.83 & & 4 & 2.2 \\
\hline $3 p 7 q$ & $\begin{array}{l}\text { Bacillus } \\
\text { thermoproteolyticus }\end{array}$ & water & 2.03 & 2.04 & 1.96 & 2.90 & 1.77 & & 4 & 2.2 \\
\hline $3 p 7 r$ & $\begin{array}{l}\text { Bacillus } \\
\text { thermoproteolyticus }\end{array}$ & & 2.01 & 2.04 & 1.97 & 2.86 & & & 3 & 2.2 \\
\hline $3 p 7 s$ & $\begin{array}{l}\text { Bacillus } \\
\text { thermoproteolyticus }\end{array}$ & & 2 & 2.06 & 1.98 & 2.80 & & & 3 & 2.2 \\
\hline $3 p 7 t$ & $\begin{array}{l}\text { Bacillus } \\
\text { thermoproteolyticus }\end{array}$ & water & 2.03 & 2.04 & 1.91 & 2.93 & 1.83 & & 4 & 2.2 \\
\hline $3 p 7 u$ & $\begin{array}{l}\text { Bacillus } \\
\text { thermoproteolyticus }\end{array}$ & water & 2.01 & 2.05 & 1.93 & 2.81 & 1.75 & & 4 & 2.2 \\
\hline $3 p 7 v$ & $\begin{array}{l}\text { Bacillus } \\
\text { thermoproteolyticus }\end{array}$ & water & 2.01 & 2.09 & 2.00 & 2.63 & 1.8 & & $4(5)$ & 2.2 \\
\hline $3 p 7 w$ & $\begin{array}{l}\text { Bacillus } \\
\text { thermoproteolyticus }\end{array}$ & water & 1.99 & 2.09 & 2.03 & 2.52 & 1.79 & & $4(5)$ & 2.2 \\
\hline 3 puu & Escherichia coli & water & 2.14 & 2.11 & 2.02 & 2.81 & 2.58 & 2.61 & $3(5)$ & 2.15 \\
\hline
\end{tabular}




\begin{tabular}{|c|c|c|c|c|c|c|c|c|c|c|}
\hline 3qgo & $\begin{array}{l}\text { Bacillus } \\
\text { thermoproteolyticus }\end{array}$ & & 2.06 & 2.04 & 1.92 & 3.05 & & & 3 & 1.45 \\
\hline 3qh1 & $\begin{array}{l}\text { Bacillus } \\
\text { thermoproteolyticus }\end{array}$ & $\begin{array}{l}\mathrm{n} \text { - } \\
\text { [(benzyloxy)carb } \\
\text { onyl]-I-aspartic } \\
\text { acid }\end{array}$ & 2.03 & 1.99 & 2.00 & 3.02 & 1.93 & & 4 & 1.55 \\
\hline 3qh5 & $\begin{array}{l}\text { Bacillus } \\
\text { thermoproteolyticus }\end{array}$ & $\begin{array}{l}\mathrm{n} \text { - } \\
\text { [(benzyloxy)carb } \\
\text { onyl]-I-aspartic } \\
\text { acid }\end{array}$ & 2 & 2.02 & 1.97 & 2.93 & 1.99 & & 4 & 1.5 \\
\hline $3 q j x$ & Escherichia coli & & 2 & 2.02 & 1.91 & 2.81 & & & 4 & 1.45 \\
\hline $3+87$ & $\begin{array}{l}\text { Bacillus } \\
\text { thermoproteolyticus }\end{array}$ & $\begin{array}{l}\text { (2s)-2-(\{n-[(r)- } \\
\text { (\{[(benzyloxy)car } \\
\text { bonyl]amino\}met } \\
\text { hyl) } \\
\text { (hydroxy)phosph } \\
\text { oryl]-l- } \\
\text { leucyl\}amino)but } \\
\text { anoic acid }\end{array}$ & 2.03 & 2.04 & 1.98 & 2.97 & 1.98 & & 4 & 1.28 \\
\hline $3 \mathrm{t} 8 \mathrm{c}$ & $\begin{array}{l}\text { Bacillus } \\
\text { thermoproteolyticus }\end{array}$ & $\begin{array}{l}\text { n-[(r)- } \\
\text { (\{[(benzyloxy)car } \\
\text { bonyl]amino } \\
\text { \}methyl)(hydroxy } \\
\text { Iphosphoryl]-I- } \\
\text { leucyl-I-norvaline }\end{array}$ & 2.06 & 2.04 & 1.98 & 2.94 & 1.97 & & 4 & 1.66 \\
\hline $3 t 8 d$ & $\begin{array}{l}\text { Bacillus } \\
\text { thermoproteolyticus }\end{array}$ & $\begin{array}{l}\text { n-[(s)- } \\
\text { (\{[(benzyloxy)car } \\
\text { bonyl]amino }\} \\
\text { methyl)(hydroxy) } \\
\text { phosphoryl]-I- } \\
\text { leucyl-I- } \\
\text { isoleucine }\end{array}$ & 2.03 & 2.04 & 1.98 & 2.95 & 1.99 & & 4 & 1.41 \\
\hline $3 \mathrm{t} 8 \mathrm{~h}$ & $\begin{array}{l}\text { Bacillus } \\
\text { thermoproteolyticus }\end{array}$ & $\begin{array}{l}\text { n-[(s)- } \\
\text { (\{[(benzyloxy)car } \\
\text { bonyl]amino } \\
\text { \}methyl)(hydroxy } \\
\text { )phosphoryl]-I- } \\
\text { leucyl-I-valine }\end{array}$ & 1.99 & 1.99 & 1.99 & 2.98 & 2.03 & & 4 & 1.45 \\
\hline 3 tli & $\begin{array}{l}\text { Bacillus } \\
\text { thermoproteolyticus }\end{array}$ & water & 2.02 & 2.02 & 2.23 & 2.27 & 1.93 & & 5 & 1.95 \\
\hline $3 \mathrm{tmn}$ & $\begin{array}{l}\text { Bacillus } \\
\text { thermoproteolyticus }\end{array}$ & water & 2.12 & 2.12 & 2.17 & 2.80 & 2.13 & & 4 & 1.7 \\
\hline $4 d 9 w$ & $\begin{array}{l}\text { Bacillus } \\
\text { thermoproteolyticus }\end{array}$ & $\begin{array}{l}\text { n-[(r)- } \\
\text { (\{[(benzyloxy)car } \\
\text { bonyl]amino }\} \\
\text { methyl)(hydroxy) } \\
\text { phosphoryl]-I- } \\
\text { leucyl-I- } \\
\text { phenylalanine }\end{array}$ & 2.02 & 2.05 & 1.98 & 2.96 & 1.99 & & 4 & 1.38 \\
\hline $4 \mathrm{~h} 57$ & $\begin{array}{l}\text { Bacillus } \\
\text { thermoproteolyticus }\end{array}$ & $\begin{array}{l}\mathrm{n}-[(\mathrm{s})- \\
\text { (\{[(benzyloxy)car } \\
\text { bonyl]amino } \\
\text { \}methyl)(hydroxy } \\
\text { Iphosphoryl]-I- } \\
\text { leucyl-I-leucine }\end{array}$ & 2.04 & 2.03 & 1.98 & 2.96 & 1.92 & & 4 & 1.56 \\
\hline $4 \mathrm{~m} 65$ & $\begin{array}{l}\text { Thermus } \\
\text { thermophilus }\end{array}$ & asparagine & 2.03 & 2.13 & 2.00 & 3.14 & 2.19 & 2.28 & $4(5)$ & 1.6 \\
\hline $4 m w p$ & $\begin{array}{l}\text { Bacillus } \\
\text { thermoproteolyticus }\end{array}$ & $\begin{array}{l}\mathrm{p}- \\
((((\text { benzyloxy)car } \\
\text { bonyl)amino)met } \\
\text { hyl)-n-((s)-4- } \\
\text { methyl- 1-oxo-1- }\end{array}$ & 2.02 & 2.03 & 1.95 & 2.96 & 1.98 & & 4 & 1.23 \\
\hline
\end{tabular}




\begin{tabular}{|c|c|c|c|c|c|c|c|c|c|c|}
\hline & & $\begin{array}{l}\text { (phenethylamino } \\
\text { Ipentan-2- } \\
\text { yl)phosphonamid } \\
\text { ic acid }\end{array}$ & & & & & & & & \\
\hline $4 m \times j$ & $\begin{array}{l}\text { Bacillus } \\
\text { thermoproteolyticus }\end{array}$ & $\begin{array}{l}\text { p- } \\
\text { ((((benzyloxy)car } \\
\text { bonyl)amino)met } \\
\text { hyl)-n-((s)-4- } \\
\text { methyl-1-oxo-1- } \\
\text { (propylamino)pe } \\
\text { ntan-2- } \\
\text { yl)phosphonamid } \\
\text { ic acid }\end{array}$ & 2.02 & 2.04 & 1.96 & 2.98 & 2 & & 4 & 1.35 \\
\hline $4 n 4 e$ & $\begin{array}{l}\text { Bacillus } \\
\text { thermoproteolyticus }\end{array}$ & $\begin{array}{l}\text { p- } \\
\text { ((((benzyloxy)car } \\
\text { bonyl)amino)met } \\
\text { hyl)-n-((s)-1- } \\
\text { ((3,3- } \\
\text { dimethylbutyl)a } \\
\text { mino)-4-methyl- } \\
\text { 1-oxopentan-2- } \\
\text { yl) } \\
\text { phosphonamidic } \\
\text { acid }\end{array}$ & 2.01 & 2.03 & 1.96 & 2.97 & 1.98 & & 4 & 1.13 \\
\hline $4 n 5 p$ & $\begin{array}{l}\text { Bacillus } \\
\text { thermoproteolyticus }\end{array}$ & & 2.03 & 2.03 & 1.96 & 2.98 & & & 4 & 1.25 \\
\hline $4 n 66$ & $\begin{array}{l}\text { Bacillus } \\
\text { thermoproteolyticus }\end{array}$ & $\begin{array}{l}\text { p- } \\
\text { ((((benzyloxy)car } \\
\text { bonyl)amino)met } \\
\text { hyl)-n-((s)-4- } \\
\text { methyl-1- } \\
\text { (neopentylamino } \\
\text { )-1-oxopentan-2- } \\
\text { yl)phosphonamid } \\
\text { ic acid }\end{array}$ & 2.02 & 2.02 & 1.96 & 2.95 & 2 & & 4 & 1.44 \\
\hline 4oi5 & $\begin{array}{l}\text { Bacillus } \\
\text { thermoproteolyticus }\end{array}$ & $\begin{array}{l}\text { p- } \\
\text { ((((benzyloxy)car } \\
\text { bonyl)amino)met } \\
\text { hyl)-n-((s)-1- } \\
\text { ((3,3- } \\
\text { dimethylbutyl)a } \\
\text { mino)-4-methyl- } \\
\text { 1-oxopentan-2- } \\
\text { yl) } \\
\text { phosphonamidic } \\
\text { acid }\end{array}$ & 2.03 & 2.02 & 1.97 & 2.96 & 2.01 & & 4 & 1.3 \\
\hline 4ow3 & $\begin{array}{l}\text { Bacillus } \\
\text { thermoproteolyticus }\end{array}$ & water & 2.06 & 1.98 & 2.47 & 2.57 & 2.08 & & $4(5)$ & 2.1 \\
\hline $4 \mathrm{tli}$ & $\begin{array}{l}\text { Bacillus } \\
\text { thermoproteolyticus }\end{array}$ & water & 2 & 2.08 & 2.24 & 2.29 & 1.77 & & $4(5)$ & 1.95 \\
\hline $4 \mathrm{t} \ln$ & $\begin{array}{l}\text { Bacillus } \\
\text { thermoproteolyticus }\end{array}$ & $\begin{array}{l}\text { I-leucyl- } \\
\text { hydroxylamine }\end{array}$ & 2.27 & 1.99 & 1.98 & 2.84 & 2.1 & 2 & 5 & 2.3 \\
\hline $4 \mathrm{tmn}$ & $\begin{array}{l}\text { Bacillus } \\
\text { thermoproteolyticus }\end{array}$ & $\begin{array}{l}\mathrm{n}-[(\mathrm{s})-[(1 \mathrm{r})-1- \\
\{[(\text { benzyloxy)carb } \\
\text { onyl]amino\}-2- } \\
\text { phenylethyl](hyd } \\
\text { roxy)phosphoryl] } \\
\text {-I-leucyl-I-alanine }\end{array}$ & 2.09 & 2.11 & 2.04 & 2.92 & 1.18 & 2.59 & $4(5)$ & 1.7 \\
\hline 4 tnl & $\begin{array}{l}\text { Bacillus } \\
\text { thermoproteolyticus }\end{array}$ & water & 2 & 2.01 & 2.16 & 2.83 & 2.03 & & 4 & 1.8 \\
\hline $5 a 3 y$ & $\begin{array}{l}\text { Bacillus } \\
\text { thermoproteolyticus }\end{array}$ & water & 2.07 & 2.06 & 2.01 & 3.09 & 1.98 & & 4 & 1.27 \\
\hline
\end{tabular}




\begin{tabular}{|c|c|c|c|c|c|c|c|c|c|c|}
\hline $5 \mathrm{dlh}$ & $\begin{array}{l}\text { Geobacillus } \\
\text { stearothermophilus }\end{array}$ & water & 2.18 & 2.09 & 2.30 & 2.45 & 2.36 & 2.59 & $5(6)$ & 2.25 \\
\hline $5 \mathrm{dpe}$ & $\begin{array}{l}\text { Bacillus } \\
\text { thermoproteolyticus }\end{array}$ & 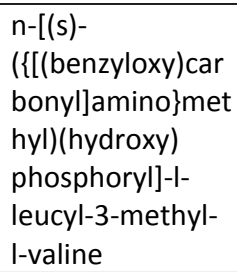 & 2.02 & 2.04 & 1.98 & 2.99 & 1.97 & & 4 & 1.34 \\
\hline $5 \mathrm{dpf}$ & $\begin{array}{l}\text { Bacillus } \\
\text { thermoproteolyticus }\end{array}$ & $\begin{array}{l}\mathrm{n}-[(\mathrm{s})- \\
\text { (\{[(benzyloxy)car} \\
\text { bonyl]amino\}met } \\
\text { hyl)(hydroxy) } \\
\text { phosphoryl]-l- } \\
\text { leucyl-4-methyl- } \\
\text { I-leucine }\end{array}$ & 2.03 & 2.04 & 2.00 & 2.96 & 1.95 & & 4 & 1.47 \\
\hline $5 \mathrm{fsj}$ & $\begin{array}{l}\text { Bacillus } \\
\text { thermoproteolyticus }\end{array}$ & & 2.03 & 2.06 & 1.98 & 3.02 & & & 3 & 1.2 \\
\hline $5 \mathrm{fss}$ & $\begin{array}{l}\text { Bacillus } \\
\text { thermoproteolyticus }\end{array}$ & & 2.03 & 2.01 & 3.17 & 4.11 & & & 3 & 1.5 \\
\hline $5 f \times n$ & $\begin{array}{l}\text { Bacillus } \\
\text { thermoproteolyticus }\end{array}$ & water & 2.06 & 2.1 & 2.12 & 2.87 & 2.18 & & 4 & 1.45 \\
\hline $5 \mathrm{js} 3$ & $\begin{array}{l}\text { Bacillus } \\
\text { thermoproteolyticus }\end{array}$ & $\begin{array}{l}\mathrm{n}^{\sim} 2^{\sim}-[(\mathrm{r})- \\
\text { (\{[(benzyloxy)car} \\
\text { bonyl]amino\}met } \\
\text { hyl) } \\
\text { (hydroxy)phosph } \\
\text { oryl]-n-[(2s)- } \\
2,3,3- \\
\text { trimethylbutyl]-I- } \\
\text { leucinamide }\end{array}$ & 2.02 & 2.02 & 1.98 & 2.97 & 1.97 & & 4 & 1.16 \\
\hline $5 \mathrm{jss}$ & $\begin{array}{l}\text { Bacillus } \\
\text { thermoproteolyticus }\end{array}$ & $\begin{array}{l}\mathrm{n}^{\sim} 2^{\sim}-[(\mathrm{r})- \\
\text { (\{[(benzyloxy)car} \\
\text { bonyl]amino\}met } \\
\text { hyl) } \\
\text { (hydroxy)phosph } \\
\text { oryl]-n-[(2r)- } \\
2,3,3- \\
\text { trimethylbutyl]-I- } \\
\text { leucinamide }\end{array}$ & 2.02 & 2.03 & 1.97 & 2.98 & 1.98 & & 4 & 1.19 \\
\hline $5 j \mathrm{jt} 9$ & $\begin{array}{l}\text { Bacillus } \\
\text { thermoproteolyticus }\end{array}$ & $\begin{array}{l}\sim\{\mathrm{n}\}-[(2 \sim\{\mathrm{s}\})-1- \\
{[[(2 \sim\{\mathrm{s}\})-2,3-} \\
\text { dimethylbutyl]a } \\
\text { mino]-4- methyl- } \\
\text { 1-oxidanylidene- } \\
\text { pentan-2-yl]- } \\
\text { (phenylmethoxyc } \\
\text { arbonylaminome } \\
\text { thyl) } \\
\text { phosphonamidic } \\
\text { acid }\end{array}$ & 2.03 & 2.04 & 1.98 & 2.97 & 1.98 & & 4 & 1.26 \\
\hline $5 j v i$ & $\begin{array}{l}\text { Bacillus } \\
\text { thermoproteolyticus }\end{array}$ & $\begin{array}{l}\{\mathrm{n}\}-[(2 \sim\{\mathrm{s}\})-1- \\
{[[(2 \sim\{\mathrm{r}\})-2,3-} \\
\text { dimethylbutyl]a } \\
\text { mino]-4- methyl- } \\
\text { 1-oxidanylidene- } \\
\text { pentan-2-yl]- } \\
\text { (phenylmethoxyc } \\
\text { arbonylaminome } \\
\text { thyl) } \\
\text { phosphonamidic } \\
\text { acid }\end{array}$ & 2.02 & 2.02 & 1.98 & 2.97 & 1.98 & & 4 & 1.12 \\
\hline
\end{tabular}




\begin{tabular}{|c|c|c|c|c|c|c|c|c|c|}
\hline $5 j x n$ & $\begin{array}{l}\text { Bacillus } \\
\text { thermoproteolyticus }\end{array}$ & $\begin{array}{l}\{n\}-[(2 \sim\{s\})-1- \\
{[[(2 \sim\{s\})-2,3-} \\
\text { dimethylbutyl]a } \\
\text { mino]-4- methyl- } \\
\text { 1-oxidanylidene- } \\
\text { pentan-2-yl]- } \\
\text { (phenylmethoxyc } \\
\text { arbonylaminome } \\
\text { thyl) } \\
\text { phosphonamidic } \\
\text { acid }\end{array}$ & 2.04 & 2.05 & 1.98 & 2.95 & 1.98 & 4 & 1.38 \\
\hline $5 \mathrm{k} 7 \mathrm{t}$ & $\begin{array}{l}\text { Bacillus } \\
\text { thermoproteolyticus }\end{array}$ & - & 2.2 & 2.19 & 2.04 & 3.07 & & 3 & 2.5 \\
\hline $513 u$ & $\begin{array}{l}\text { Bacillus } \\
\text { thermoproteolyticus }\end{array}$ & $\begin{array}{l}\mathrm{n}^{\sim} 2^{\sim-}[(\mathrm{r})- \\
(\{[(\text { benzyloxy)car } \\
\text { bonyl]amino\}met } \\
\text { hyl) } \\
\text { (hydroxy)phosph } \\
\text { oryl]-n-[(2r)- } \\
2,3,3- \\
\text { trimethylbutyl]-I- } \\
\text { leucinamide }\end{array}$ & 2.03 & 2.03 & 1.99 & 2.98 & 1.99 & 4 & 1.23 \\
\hline 5141 & $\begin{array}{l}\text { Bacillus } \\
\text { thermoproteolyticus }\end{array}$ & $\begin{array}{l}\{\mathrm{n}\}-[(2 \sim\{\mathrm{s}\})-1- \\
{[[(2 \sim\{r\})-2,3-} \\
\text { dimethylbutyl]a } \\
\text { mino]-4- methyl- } \\
\text { 1-oxidanylidene- } \\
\text { pentan-2-yl]- } \\
\text { (phenylmethoxyc } \\
\text { arbonylaminome } \\
\text { thyl)phosphona } \\
\text { midic acid }\end{array}$ & 2.02 & 2.01 & 1.98 & 2.98 & 1.98 & 4 & 1.25 \\
\hline $518 p$ & $\begin{array}{l}\text { Bacillus } \\
\text { thermoproteolyticus }\end{array}$ & $\begin{array}{l}\mathrm{n}^{\sim} 2^{\sim}-[(\mathrm{r})- \\
(\{[(\text { benzyloxy)car } \\
\text { bonyl]amino\}met } \\
\text { hyl) } \\
\text { (hydroxy)phosph } \\
\text { oryl]-n-[(2s)- } \\
2,3,3- \\
\text { trimethylbutyl]-I- } \\
\text { leucinamide }\end{array}$ & 2.02 & 2.02 & 1.98 & 2.97 & 1.96 & 4 & 1.29 \\
\hline 5lif & $\begin{array}{l}\text { Bacillus } \\
\text { thermoproteolyticus }\end{array}$ & $\begin{array}{l}(2 \sim\{s\})-3- \\
\text { cyclohexyl-2- } \\
{\left[\left[\left(2^{\sim}\{\mathrm{s}\}\right)-4-\right.\right.} \\
\text { methyl-2- } \\
\text { [[oxidanyl } \\
\text { (phenylmethoxyc } \\
\text { arbonylaminome } \\
\text { thyl) } \\
\text { phosphoryl]amin } \\
\text { o]pentanoyl] } \\
\text { amino]propanoic } \\
\text { acid }\end{array}$ & 2.02 & 2.02 & 1.96 & 2.94 & 1.95 & 4 & 1.31 \\
\hline $5 \operatorname{lvd}$ & $\begin{array}{l}\text { Bacillus } \\
\text { thermoproteolyticus }\end{array}$ & $\begin{array}{l}\text { (2 \{s\})-4-methyl- } \\
2-\left[\left[\left(2^{\sim}\{\mathrm{s}\}\right)-3-\right.\right. \\
\text { oxidanyl-2- } \\
\text { [[oxidanyl } \\
\text { (phenylmethoxyc } \\
\text { arbonylaminome } \\
\text { thyl) } \\
\text { phosphoryl]amin } \\
\text { o]propanoyl] }\end{array}$ & 2.01 & 2.02 & 1.97 & 2.96 & 1.98 & 4 & 1.25 \\
\hline
\end{tabular}




\begin{tabular}{|c|c|c|c|c|c|c|c|c|c|}
\hline & & $\begin{array}{l}\text { amino]pentanoic } \\
\text { acid }\end{array}$ & & & & & & & \\
\hline $51 w d$ & $\begin{array}{l}\text { Bacillus } \\
\text { thermoproteolyticus }\end{array}$ & jc96 & 2.01 & 2.02 & 1.98 & 2.98 & 2 & 4 & 1.23 \\
\hline $5 \mathrm{~m} 5 f$ & $\begin{array}{l}\text { Bacillus } \\
\text { thermoproteolyticus }\end{array}$ & $\begin{array}{l}\text { (2 \{s\})-4-methyl- } \\
2-[2-[[o x i d a n y l \\
\text { (phenylmethoxyc } \\
\text { arbonylaminome } \\
\text { thyl) } \\
\text { phosphoryl]amin } \\
\text { o]ethanoylamino } \\
\text { ] pentanoic acid }\end{array}$ & 2.02 & 2.02 & 1.98 & 2.98 & 1.96 & 4 & 1.33 \\
\hline $5 \mathrm{~m} 69$ & $\begin{array}{l}\text { Bacillus } \\
\text { thermoproteolyticus }\end{array}$ & $\begin{array}{l}\text { (2 \{s\})-4-methyl- } \\
2-[2-[[o x i d a n y l \\
\text { (phenylmethoxyc } \\
\text { arbonylaminome } \\
\text { thyl) } \\
\text { phosphoryl]amin } \\
\text { o]ethanoylamino } \\
\text { ] pentanoic acid }\end{array}$ & 2 & 2.03 & 1.99 & 2.96 & 1.97 & 4 & 1.44 \\
\hline $5 \mathrm{~m} 9 \mathrm{w}$ & $\begin{array}{l}\text { Bacillus } \\
\text { thermoproteolyticus }\end{array}$ & $\begin{array}{l}\text { (2 \{s\})-4-methyl- } \\
2-[2-[[o x i d a n y l \\
\text { (phenylmethoxyc } \\
\text { arbonylaminome } \\
\text { thyl) } \\
\text { phosphoryl]amin } \\
\text { o]ethanoylamino } \\
\text { ] pentanoic acid }\end{array}$ & 2.01 & 2.01 & 1.98 & 2.97 & 1.96 & 4 & 1.21 \\
\hline $5 \mathrm{ma7}$ & $\begin{array}{l}\text { Bacillus } \\
\text { thermoproteolyticus }\end{array}$ & $\begin{array}{l}(2 \sim\{s\})-2- \\
{[[(2 \sim\{s\})-3-} \\
\text { azanyl-2- } \\
\text { [[oxidanyl } \\
\text { (phenylmethoxyc } \\
\text { arbonylaminome } \\
\text { thyl) } \\
\text { phosphoryl]amin } \\
\text { o]propanoyl] } \\
\text { amino]-4- } \\
\text { methyl- } \\
\text { pentanoic acid }\end{array}$ & 2.01 & 2.03 & 1.99 & 2.99 & 1.97 & 4 & 1.3 \\
\hline $5 \mathrm{mnr}$ & $\begin{array}{l}\text { Bacillus } \\
\text { thermoproteolyticus }\end{array}$ & $\begin{array}{l}\text { (2 \{s\})-4-azanyl- } \\
2-\left[\left[\left(2^{\sim}\{s\}\right)-4-\right.\right. \\
\text { methyl-2- } \\
\text { [[oxidanyl } \\
\text { (phenylmethoxyc } \\
\text { arbonylaminome } \\
\text { thyl) } \\
\text { phosphoryl]amin } \\
\text { o]pentanoyl] } \\
\text { amino]butanoic } \\
\text { acid }\end{array}$ & 2.02 & 2.03 & 1.99 & 2.97 & 1.99 & 4 & 1.25 \\
\hline $5 \mathrm{n} 2 \mathrm{t}$ & $\begin{array}{l}\text { Bacillus } \\
\text { thermoproteolyticus }\end{array}$ & $\begin{array}{l}\{\mathrm{n}\}-[(2 \sim\{s\})-1-(3- \\
\text { azanylpropylami } \\
\text { no)-4-methyl-1- } \\
\text { oxidanylidene- } \\
\text { pentan-2-yl]- } \\
\text { (phenylmethoxyc } \\
\text { arbonylaminome } \\
\text { thyl) } \\
\text { phosphonamidic } \\
\text { acid }\end{array}$ & 2.04 & 2.04 & 1.99 & 2.97 & 1.97 & 4 & 1.38 \\
\hline $5 n 2 x$ & $\begin{array}{l}\text { Bacillus } \\
\text { thermoproteolyticus }\end{array}$ & $\begin{array}{l}\text { (2 \{s\})-5-azanyl- } \\
2-[[(2 \sim\{s\})-4-\end{array}$ & 2.03 & 2.04 & 1.99 & 2.98 & 1.97 & 4 & 1.21 \\
\hline
\end{tabular}




\begin{tabular}{|c|c|c|c|c|c|c|c|c|c|c|}
\hline & & $\begin{array}{l}\text { methyl-2- } \\
\text { [[oxidanyl(phenyl } \\
\text { methoxycarbonyl } \\
\text { aminomethyl) } \\
\text { phosphoryl]amin } \\
\text { o]pentanoyl]ami } \\
\text { no]pentanoic } \\
\text { acid }\end{array}$ & & & & & & & & \\
\hline $5 n 2 z$ & $\begin{array}{l}\text { Bacillus } \\
\text { thermoproteolyticus }\end{array}$ & $\begin{array}{l}\text { \{n\}-[(2 \{s\})-1-(4- } \\
\text { azanylbutylamin } \\
\text { o)-4-methyl-1- } \\
\text { oxidanylidene- } \\
\text { pentan-2-yl]- } \\
\text { (phenylmethoxyc } \\
\text { arbonylaminome } \\
\text { thyl)phosphona } \\
\text { midic acid }\end{array}$ & 2.03 & 2.04 & 2.00 & 2.98 & 1.97 & & 4 & 1.37 \\
\hline $5 n 31$ & $\begin{array}{l}\text { Bacillus } \\
\text { thermoproteolyticus }\end{array}$ & $\begin{array}{l}(2 \sim\{s\})-6-\text {-azanyl- } \\
2 \text {-[[(2 }\{s\})-4- \\
\text { methyl-2- } \\
\text { [[oxidanyl(phenyl } \\
\text { methoxycarbonyl } \\
\text { aminomethyl) } \\
\text { phosphoryl]amin } \\
\text { o]pentanoyl]ami } \\
\text { no]hexanoic acid }\end{array}$ & 2.04 & 2.05 & 2.00 & 2.97 & 1.97 & & 4 & 1.37 \\
\hline $5 n 34$ & $\begin{array}{l}\text { Bacillus } \\
\text { thermoproteolyticus }\end{array}$ & $\begin{array}{l}\{\mathrm{n}\}-\left[\left(2^{\sim}\{\mathrm{s}\}\right)-1-(2-\right. \\
\text { azanylethylamin } \\
\text { o)-4-methyl-1- } \\
\text { oxidanylidene- } \\
\text { pentan-2-yl]- } \\
\text { (phenylmethoxyc } \\
\text { arbonylaminome } \\
\text { thyl) } \\
\text { phosphonamidic } \\
\text { acid }\end{array}$ & 2.02 & 2.02 & 1.99 & 2.96 & 1.96 & & 4 & 1.22 \\
\hline $5 n 3 v$ & $\begin{array}{l}\text { Bacillus } \\
\text { thermoproteolyticus }\end{array}$ & $\begin{array}{l}\text { \{n\}-[(2 \{s\})-1- } \\
\text { (aminomethylam } \\
\text { ino)-4-methyl-1- } \\
\text { 5n3v.pdb:hetna } \\
\text { m } 2815 \\
\text { oxidanylidene- } \\
\text { pentan-2-yl]- } \\
\text { (phenylmethoxyc } \\
\text { arbonylaminome } \\
\text { thyl) } \\
\text { phosphonamidic } \\
\text { acid;hydrogen }\end{array}$ & 2.02 & 2.03 & 1.98 & 2.97 & 1.98 & & 4 & 1.12 \\
\hline $5 n 3 y$ & $\begin{array}{l}\text { Bacillus } \\
\text { thermoproteolyticus }\end{array}$ & $\begin{array}{l}\text { (2 \{s\}\})-3-azanyl- } \\
2 \text {-[[(2 }\{\{\}\})-4- \\
\text { methyl-2- } \\
\text { [[oxidanyl } \\
\text { (phenylmethoxyc } \\
\text { arbonylaminome } \\
\text { thyl) } \\
\text { phosphoryl]amin } \\
\text { o]pentanoyl]ami } \\
\text { no] propanoic } \\
\text { acid }\end{array}$ & 2.02 & 2.06 & 1.99 & 2.96 & 1.98 & & 4 & 1.34 \\
\hline 5onr & $\begin{array}{l}\text { Bacillus } \\
\text { thermoproteolyticus }\end{array}$ & water & 1.98 & 2.08 & 2.01 & 3.06 & 2.11 & 2.41 & 5 & 1.39 \\
\hline $5+9 i$ & $\begin{array}{l}\text { Bacillus } \\
\text { thermoproteolyticus }\end{array}$ & water & 2.08 & 2.05 & 2.16 & 2.29 & 2.05 & & 5 & 2.09 \\
\hline
\end{tabular}




\begin{tabular}{|c|c|c|c|c|c|c|c|c|c|c|}
\hline $5 \mathrm{t} 9 \mathrm{k}$ & $\begin{array}{l}\text { Bacillus } \\
\text { thermoproteolyticus }\end{array}$ & water & 2.12 & 2.06 & 2.18 & 2.26 & 2.11 & 2.17 & 6 & 2.1 \\
\hline $5 t 9 q$ & $\begin{array}{l}\text { Bacillus } \\
\text { thermoproteolyticus }\end{array}$ & phospate ion & 2.12 & 2.07 & 2.20 & 2.30 & 1.82 & & 5 & 2.1 \\
\hline 5 tac & $\begin{array}{l}\text { Bacillus } \\
\text { thermoproteolyticus }\end{array}$ & water & 2.09 & 2.04 & 2.20 & 3.50 & 1.97 & 2.6 & 5 & 2.04 \\
\hline 5 tad & $\begin{array}{l}\text { Bacillus } \\
\text { thermoproteolyticus }\end{array}$ & phospate ion & 2.09 & 2.03 & 2.02 & 2.98 & 1.81 & & 4 & 2.09 \\
\hline 5tae & $\begin{array}{l}\text { Bacillus } \\
\text { thermoproteolyticus }\end{array}$ & phospate ion & 2.08 & 2.06 & 2.15 & 2.20 & 1.97 & & 5 & 2.3 \\
\hline 5 tai & $\begin{array}{l}\text { Bacillus } \\
\text { thermoproteolyticus }\end{array}$ & water & 2.07 & 2.11 & 2.04 & 2.87 & 1.17 & 2.12 & 5 & 2.3 \\
\hline 5taj & $\begin{array}{l}\text { Bacillus } \\
\text { thermoproteolyticus }\end{array}$ & water & 2.08 & 2.07 & 2.19 & 3.09 & 2.16 & & 5 & 2.03 \\
\hline 5tak & $\begin{array}{l}\text { Bacillus } \\
\text { thermoproteolyticus }\end{array}$ & water & 2.07 & 2.12 & 2.11 & 2.28 & 2.08 & & 5 & 2 \\
\hline $5 \mathrm{tli}$ & $\begin{array}{l}\text { Bacillus } \\
\text { thermoproteolyticus }\end{array}$ & water & 2.01 & 2.02 & 2.23 & 2.30 & 2.08 & & 5 & 2.1 \\
\hline $5 \mathrm{t} / \mathrm{n}$ & $\begin{array}{l}\text { Bacillus } \\
\text { thermoproteolyticus }\end{array}$ & $\begin{array}{l}\text { honh- } \\
\text { benzylmalonyl-I- } \\
\text { alanylglycine-p- } \\
\text { nitroanilide }\end{array}$ & 2.19 & 1.96 & 2.11 & 2.94 & 2.1 & 2.26 & 5 & 2.3 \\
\hline $5 \mathrm{tmn}$ & $\begin{array}{l}\text { Bacillus } \\
\text { thermoproteolyticus }\end{array}$ & $\begin{array}{l}\mathrm{n} \text {-[(s)- } \\
\text { (\{[(benzyloxy)car } \\
\text { bonyl]amino\}met } \\
\text { hyl)(hydroxy) } \\
\text { phosphoryl]--- } \\
\text { leucyl-I-leucine }\end{array}$ & 2.04 & 2.09 & 2.07 & 2.86 & 2.08 & & 4 & 1.6 \\
\hline $5 w r 2$ & $\begin{array}{l}\text { Geobacillus } \\
\text { stearothermophilus }\end{array}$ & $\begin{array}{l}\text { n- } \\
\text { [(benzyloxy)carb } \\
\text { onyl]-l-aspartic } \\
\text { acid }\end{array}$ & 2.04 & 2.06 & 1.90 & 2.91 & 1.98 & 2.67 & $4(5)$ & 2 \\
\hline $5 w r 3$ & $\begin{array}{l}\text { Geobacillus } \\
\text { stearothermophilus }\end{array}$ & $\begin{array}{l}\mathrm{n} \text { - } \\
\text { [(benzyloxy)carb } \\
\text { onyl]-I-aspartic } \\
\text { acid }\end{array}$ & 2.07 & 2.03 & 1.98 & 2.99 & 1.96 & 2.63 & $4(5)$ & 2.1 \\
\hline $5 w r 4$ & $\begin{array}{l}\text { Geobacillus } \\
\text { stearothermophilus }\end{array}$ & water & 2.09 & 2.05 & 2.04 & 2.84 & 2.11 & & 4 & 2.1 \\
\hline $5 w r 5$ & $\begin{array}{l}\text { Geobacillus } \\
\text { stearothermophilus }\end{array}$ & $\begin{array}{l}\mathrm{n}- \\
\text { [(benzyloxy)carb } \\
\text { onyl]-I-aspartic } \\
\text { acid }\end{array}$ & 2.02 & 2.1 & 1.85 & 2.94 & 2.02 & & 4 & 1.9 \\
\hline $5 w r 6$ & $\begin{array}{l}\text { Geobacillus } \\
\text { stearothermophilus }\end{array}$ & $\begin{array}{l}\mathrm{n}- \\
\text { [(benzyloxy)carb } \\
\text { onyl]-I-aspartic } \\
\text { acid }\end{array}$ & 1.99 & 2.11 & 1.92 & 2.93 & 2.03 & 2.62 & $4(5)$ & 2.3 \\
\hline $6 d 5 n$ & $\begin{array}{l}\text { Bacillus } \\
\text { thermoproteolyticus }\end{array}$ & water & 2.05 & 2.06 & 1.95 & 2.97 & 2.01 & & 4 & 2 \\
\hline $6 \mathrm{~d} 5 \mathrm{o}$ & $\begin{array}{l}\text { Bacillus } \\
\text { thermoproteolyticus }\end{array}$ & water & 2.09 & 2.06 & 2.03 & 2.82 & 2.65 & & 5 & 2 \\
\hline $6 d 5 p$ & $\begin{array}{l}\text { Bacillus } \\
\text { thermoproteolyticus }\end{array}$ & water & 2.1 & 2.26 & 1.83 & 3.13 & 2.26 & & 4 & 3 \\
\hline $6 d 5 q$ & $\begin{array}{l}\text { Bacillus } \\
\text { thermoproteolyticus }\end{array}$ & water & 2.17 & 2.15 & 2.01 & 2.94 & 1.97 & & 4 & 2 \\
\hline $6 \mathrm{~d} 5 \mathrm{r}$ & $\begin{array}{l}\text { Bacillus } \\
\text { thermoproteolyticus }\end{array}$ & water & 2.07 & 2.07 & 2.07 & 2.83 & 1.96 & & 4 & 2 \\
\hline $6 \mathrm{~d} 5 \mathrm{~s}$ & $\begin{array}{l}\text { Bacillus } \\
\text { thermoproteolyticus }\end{array}$ & water & 2.13 & 2.07 & 2.04 & 2.63 & 1.84 & & 5 & 2 \\
\hline $6 \mathrm{~d} 5 \mathrm{t}$ & $\begin{array}{l}\text { Bacillus } \\
\text { thermoproteolyticus }\end{array}$ & water & 2.04 & 2.05 & 1.92 & 2.98 & 2.41 & & 4 & 2 \\
\hline $6 \mathrm{~d} 5 \mathrm{u}$ & $\begin{array}{l}\text { Bacillus } \\
\text { thermoproteolyticus }\end{array}$ & water & 2.15 & 2.05 & 1.88 & 2.95 & 2.23 & 2.35 & 5 & 2 \\
\hline
\end{tabular}




\begin{tabular}{|c|c|c|c|c|c|c|c|c|c|}
\hline $6 \mathrm{fj} 2$ & $\begin{array}{l}\text { Bacillus } \\
\text { thermoproteolyticus }\end{array}$ & water & 2.03 & 2.03 & 1.99 & 3.02 & 2.07 & 4 & 1.43 \\
\hline $6 \mathrm{fsm}$ & $\begin{array}{l}\text { Geobacillus } \\
\text { stearothermophilus }\end{array}$ & phospate ion & 2.01 & 2.02 & 1.95 & 3.02 & 1.96 & 4 & 1.39 \\
\hline $6 i g 7$ & $\begin{array}{l}\text { Bacillus } \\
\text { thermoproteolyticus }\end{array}$ & & 2.03 & 2.03 & 1.97 & 2.88 & & 3 & 1.8 \\
\hline $6 \mathrm{tli}$ & $\begin{array}{l}\text { Bacillus } \\
\text { thermoproteolyticus }\end{array}$ & water & 2.02 & 2.02 & 2.22 & 2.34 & 2.02 & 5 & 2.1 \\
\hline $6 \mathrm{tmn}$ & $\begin{array}{l}\text { Bacillus } \\
\text { thermoproteolyticus }\end{array}$ & $\begin{array}{l}\mathrm{n} \text {-[(2r,4s)-4- } \\
\text { hydroxy-2-(2- } \\
\text { methylpropyl)-4- } \\
\text { oxido-7-oxo- 9- } \\
\text { phenyl-3,8- } \\
\text { dioxa-6-aza-4- } \\
\text { phosphanonan- } \\
\text { 1-oyl]-I- leucine }\end{array}$ & 2.09 & 2.1 & 1.99 & 2.89 & 2.07 & 5 & 1.6 \\
\hline $7 \mathrm{tli}$ & $\begin{array}{l}\text { Bacillus } \\
\text { thermoproteolyticus }\end{array}$ & water & 2.04 & 2.03 & 2.08 & 2.82 & 2.67 & 4 & 1.95 \\
\hline $7 \mathrm{t} \ln$ & $\begin{array}{l}\text { Bacillus } \\
\text { thermoproteolyticus }\end{array}$ & $\begin{array}{l}\text { 2-(acetyl- } \\
\text { hydroxy-amino)- } \\
\text { 4-methyl- } \\
\text { pentanoic acid } \\
\text { methyl ester }\end{array}$ & 2.1 & 2.16 & 1.91 & 2.73 & 2.48 & 4 & 2.3 \\
\hline $8 \mathrm{tli}$ & $\begin{array}{l}\text { Bacillus } \\
\text { thermoproteolyticus }\end{array}$ & water & 2.02 & 2.04 & 2.12 & 2.36 & 2.02 & 5 & 2.2 \\
\hline $8 \mathrm{t} / \mathrm{n}$ & $\begin{array}{l}\text { Bacillus } \\
\text { thermoproteolyticus }\end{array}$ & water & 1.97 & 1.93 & 1.88 & 2.79 & 2.16 & 4 & 1.6 \\
\hline
\end{tabular}

\section{Neprilysin}

\begin{tabular}{|c|c|c|c|c|c|c|c|c|c|}
\hline PDB & organism & ligand & $\mathrm{Zn}-\mathrm{H}$ & $\mathrm{Zn}-\mathrm{H}$ & $\begin{array}{l}\text { Zn-E(O) } \\
\text { closer }\end{array}$ & $\begin{array}{l}\mathrm{Zn-} \\
E(0)\end{array}$ & $\begin{array}{l}\text { Zn-ligand } \\
\text { closest }\end{array}$ & $\mathrm{CN}$ & $\begin{array}{l}\text { Resolu- } \\
\text { tion/Å }\end{array}$ \\
\hline 6gid & Human & $\begin{array}{l}\text { Phosphate ion } \\
\mathrm{PO}_{4}{ }^{3-}\end{array}$ & 2.02 & 2.10 & 1.93 & 2.77 & $\begin{array}{l}2.070 \\
2.360\end{array}$ & 5 & 1.9 \\
\hline $\begin{array}{l}5 \mathrm{v} 48 \\
\text { (chain a) }\end{array}$ & Rabbit & thiorphan & 2.03 & 2.04 & 2.00 & 2.02 & $2.08 \mathrm{~S}$ & 5 & 2.99 \\
\hline $\begin{array}{l}\text { 5v48 } \\
\text { (chain b) }\end{array}$ & Rabbit & thiorphan & 2.03 & 2.04 & 2.00 & 2.01 & $2.05 \mathrm{~S}$ & 5 & 2.99 \\
\hline $\begin{array}{l}\text { 5jmy } \\
\text { (chain a) }\end{array}$ & Human & LBQ657 & 1.94 & 1.95 & 1.88 & 2.87 & 2.080 & 4 & 2 \\
\hline $\begin{array}{l}\text { 5jmy } \\
\text { (chain b) }\end{array}$ & Human & LBQ657 & 1.97 & 1.93 & 1.95 & 2.88 & $\begin{array}{l}1.960 \\
2.610\end{array}$ & $4 / 5$ & 2 \\
\hline $4 \mathrm{zr5}$ & Rabbit & phosphoramidon & 2.10 & 2.10 & 2.09 & 2.74 & 2.050 & 4 & 2.80 \\
\hline
\end{tabular}




\begin{tabular}{|c|c|c|c|c|c|c|c|c|c|}
\hline $4 \times b h$ & Rabbit & $\mathrm{PO}^{3-}$ & 2.10 & 2.05 & 2.02 & 2.92 & 2.220 & 4 & 2.11 \\
\hline 4cth & Human & phosphoramidon & 1.98 & 1.97 & 1.95 & 2.73 & 1.960 & 4 & 2.15 \\
\hline $1 \mathrm{dmt}$ & Human & phosphoramidon & 2.03 & 2.01 & 1.96 & 2.78 & 1.930 & 4 & 2.1 \\
\hline $1 \mathrm{r} 1 \mathrm{~h}$ & Human & Phospho... & 1.97 & 2.12 & 1.98 & 2.78 & 1.940 & 4 & 1.95 \\
\hline $1 \mathrm{r} 1 \mathrm{i}$ & Human & $\begin{array}{l}\text { [2(r,s)-2-sulfanylheptanoyl]- } \\
\text { Phe-Ala }\end{array}$ & 1.71 & 2.06 & 1.85 & 2.51 & $\begin{array}{l}1.84 \mathrm{O} \\
2.10 \mathrm{~S}\end{array}$ & 6 & 2.6 \\
\hline $1 \mathrm{r} 1 \mathrm{j}$ & Human & $\begin{array}{l}\text { [2(r,s)-2-sulfanylheptanoyl]- } \\
\text { Phe-Ala }\end{array}$ & 1.97 & 2.04 & 2.01 & 2.71 & $\begin{array}{l}1.79 \mathrm{O} \\
2.39 \mathrm{~S}\end{array}$ & 6 & 2.35 \\
\hline $1 y 8 j$ & Human & $\begin{array}{l}\text { imidazo[4,5-c]pyridine } \\
\text { inhibitor }\end{array}$ & 1.90 & 2.05 & 2.08 & 2.82 & $2.20 \mathrm{~S}$ & 4 & 2.25 \\
\hline 2qpj & Human & $\begin{array}{l}\text { bifunctional NEP/DPP IV } \\
\text { inhibitor }\end{array}$ & 1.93 & 2.14 & 2.06 & 2.93 & 2.070 & 4 & 2.05 \\
\hline $2 y b 9$ & Human & heteroarylalanine diacid & 2.02 & 2.02 & 1.94 & 2.97 & $\begin{array}{l}2.060 \\
2.630\end{array}$ & 4 & 2.4 \\
\hline
\end{tabular}


Table S2. RESP calculated charge distribution, atom name and atom type for atoms within amino acids coordinating the zinc ion in DPP III crystal structures. These charges were used in human DPP III MD simulations utilizing different hybrid bonded/nonbonded models. For comparison, charges from Amber ff12sb force field and M1 model are presented. Highlighted in yellow are the atoms directly coordinated to the zinc ion (i.e. first-coordination shell atoms).

\begin{tabular}{|c|c|c|c|c|c|c|c|c|c|c|c|c|}
\hline $\begin{array}{c}\text { Param- } \\
\text { eters }\end{array}$ & $\begin{array}{l}\text { Residu } \\
\text { e name }\end{array}$ & $\begin{array}{l}\text { Atom } \\
\text { name }\end{array}$ & $\begin{array}{c}\text { Atom } \\
\text { type }\end{array}$ & Charge & $\begin{array}{c}\text { Residue } \\
\text { name }\end{array}$ & $\begin{array}{l}\text { Atom } \\
\text { name }\end{array}$ & $\begin{array}{c}\text { Atom } \\
\text { type }\end{array}$ & Charge & $\begin{array}{l}\text { Residu } \\
\text { e name }\end{array}$ & $\begin{array}{l}\text { Atom } \\
\text { name }\end{array}$ & $\begin{array}{c}\text { Atom } \\
\text { type }\end{array}$ & Charge \\
\hline \multirow{17}{*}{ 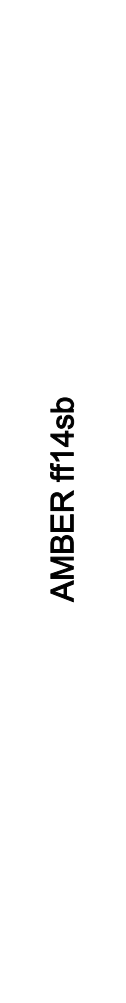 } & $\begin{array}{c}\text { GLU } \\
508\end{array}$ & $\mathrm{~N}$ & $\mathrm{~N}$ & -0.5163 & $\begin{array}{c}\text { HID } \\
450 / 455\end{array}$ & $\mathrm{~N}$ & $\mathrm{~N}$ & -0.4157 & ZN 727 & ZN & ZN & 2 \\
\hline & & $\mathrm{H}$ & $\mathrm{H}$ & 0.2936 & & $\mathrm{H}$ & $\mathrm{H}$ & 0.2719 & & & & \\
\hline & & CA & $C X$ & 0.0397 & & CA & $c x$ & 0.0188 & & & & \\
\hline & & $\mathrm{HA}$ & $\mathrm{H} 1$ & 0.1105 & & $\mathrm{HA}$ & $\mathrm{H} 1$ & 0.0881 & & & & \\
\hline & & CB & $2 C$ & 0.056 & & CB & CT & -0.0462 & & & & \\
\hline & & HB2 & $\mathrm{HC}$ & -0.0173 & & HB2 & $\mathrm{HC}$ & 0.0402 & & & & \\
\hline & & HB3 & $\mathrm{HC}$ & -0.0173 & & HB3 & $\mathrm{HC}$ & 0.0402 & & & & \\
\hline & & CG & $2 \mathrm{C}$ & 0.0136 & & CG & CC & -0.0266 & & & & \\
\hline & & HG2 & $\mathrm{HC}$ & -0.0425 & & ND1 & NA & -0.3811 & & & & \\
\hline & & HG3 & $\mathrm{HC}$ & -0.0425 & & HD1 & $\mathrm{H}$ & 0.3649 & & & & \\
\hline & & $C D$ & $\mathrm{CO}$ & 0.8054 & & CE1 & CR & 0.2057 & & & & \\
\hline & & OE1 & $\mathrm{O} 2$ & -0.8188 & & HE1 & H5 & 0.1392 & & & & \\
\hline & & OE2 & O2 & -0.8188 & & NE2 & NB & -0.5727 & & & & \\
\hline & & C & C & 0.5366 & & CD2 & CV & 0.1292 & & & & \\
\hline & & 0 & 0 & -0.5819 & & HD2 & $\mathrm{H} 4$ & 0.1147 & & & & \\
\hline & & & & & & C & C & 0.5973 & & & & \\
\hline & & & & & & 0 & 0 & -0.5679 & & & & \\
\hline \multirow{16}{*}{$\begin{array}{l}\vec{U} \\
\text { Оू } \\
\sum \\
\Sigma\end{array}$} & GL1 & $\mathrm{N}$ & $\mathrm{N}$ & -0.516 & HD1 496 & $\mathrm{~N}$ & $\mathrm{~N}$ & -0.416 & HD2 & $\mathrm{N}$ & $\mathrm{N}$ & -0.416 \\
\hline & & $\mathrm{H}$ & $\mathrm{H}$ & 0.294 & & $\mathrm{H}$ & $\mathrm{H}$ & 0.272 & & $\mathrm{H}$ & $\mathrm{H}$ & 0.272 \\
\hline & & CA & CT & 0.04 & & CA & CT & 0.019 & & CA & CT & 0.019 \\
\hline & & $\mathrm{HA}$ & $\mathrm{H} 1$ & 0.111 & & HA & $\mathrm{H} 1$ & 0.088 & & $\mathrm{HA}$ & $\mathrm{H} 1$ & 0.088 \\
\hline & & CB & CT & 0.695 & & CB & CT & 0.353 & & CB & CT & 0.137 \\
\hline & & HB2 & $\mathrm{HC}$ & -0.196 & & HB2 & $\mathrm{HC}$ & -0.046 & & HB2 & $\mathrm{HC}$ & 0.008 \\
\hline & & HB3 & $\mathrm{HC}$ & -0.196 & & HB3 & $\mathrm{HC}$ & -0.046 & & HB3 & $\mathrm{HC}$ & 0.008 \\
\hline & & CG & $\mathrm{CT}$ & -0.047 & & CG & $\mathrm{CC}$ & -0.157 & & CG & CC & 0.024 \\
\hline & & HG2 & $\mathrm{HC}$ & -0.02 & & ND1 & NA & -0.13 & & ND1 & NA & -0.076 \\
\hline & & HG3 & $\mathrm{HC}$ & -0.02 & & HD1 & $\mathrm{H}$ & 0.338 & & HD1 & $\mathrm{H}$ & 0.284 \\
\hline & & $C D$ & C & 0.754 & & CE1 & CR & 0.028 & & CE1 & CR & 0.047 \\
\hline & & OE1 & $\mathrm{OB}$ & -0.835 & & HE1 & H5 & 0.229 & & HE1 & H5 & 0.156 \\
\hline & & OE2 & $\mathrm{OB}$ & -0.661 & & NE2 & NT & -0.388 & & NE2 & NT & -0.359 \\
\hline & & C & C & 0.537 & & CD2 & CV & -0.008 & & CD2 & CV & -0.11 \\
\hline & & 0 & 0 & -0.582 & & HD2 & $\mathrm{H} 4$ & 0.146 & & HD2 & $\mathrm{H} 4$ & 0.105 \\
\hline & & & & & & C & C & 0.597 & & C & C & 0.597 \\
\hline
\end{tabular}




\begin{tabular}{|c|c|c|c|c|c|c|c|c|c|c|c|c|}
\hline & $\begin{array}{l}\text { ZN1 } \\
1085\end{array}$ & ZN & ZN1 & 1.115 & & O & $\mathrm{O}$ & -0.568 & & O & O & -0.568 \\
\hline \multirow{17}{*}{ 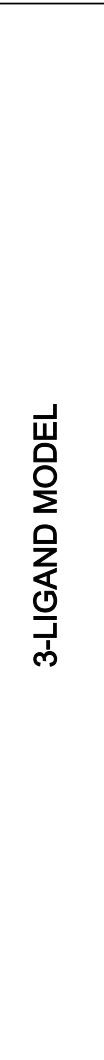 } & $\begin{array}{c}\text { GU1 } \\
508\end{array}$ & N & $N$ & -0.5163 & HD1 450 & $N$ & $N$ & -0.4157 & $\begin{array}{l}\mathrm{HD} 2 \\
455\end{array}$ & $\mathrm{~N}$ & $\mathrm{~N}$ & -0.4157 \\
\hline & & $\mathrm{H}$ & $\mathrm{H}$ & 0.352211 & & $\mathrm{H}$ & $\mathrm{H}$ & 0.303872 & & $\mathrm{H}$ & $\mathrm{H}$ & 0.41444 \\
\hline & & CA & $c x$ & 0.0397 & & $C A$ & $C X$ & 0.0188 & & CA & $c x$ & 0.0188 \\
\hline & & HA & $\mathrm{H} 1$ & 0.167786 & & $\mathrm{HA}$ & $\mathrm{H} 1$ & 0.140979 & & $\mathrm{HA}$ & $\mathrm{H} 1$ & 0.059871 \\
\hline & & $\mathrm{CB}$ & $2 \mathrm{C}$ & 0.144774 & & $\mathrm{CB}$ & CT & -0.15184 & & СB & CT & -0.06259 \\
\hline & & HB2 & $\mathrm{HC}$ & -0.01368 & & HB2 & $\mathrm{HC}$ & 0.083136 & & HB2 & $\mathrm{HC}$ & 0.035264 \\
\hline & & HB3 & $\mathrm{HC}$ & -0.01368 & & HB3 & $\mathrm{HC}$ & 0.083136 & & HB3 & $\mathrm{HC}$ & 0.035264 \\
\hline & & CG & $2 \mathrm{C}$ & 0.00257 & & CG & CC & -0.02962 & & $C G$ & $\mathrm{CC}$ & 0.030961 \\
\hline & & HG2 & $\mathrm{HC}$ & -0.00708 & & ND1 & NA & -0.0913 & & ND1 & NA & -0.09983 \\
\hline & & HG3 & $\mathrm{Hc}$ & -0.00708 & & HD1 & $\mathrm{H}$ & 0.336212 & & HD1 & $\mathrm{H}$ & 0.342661 \\
\hline & & $C D$ & $\mathrm{CO}$ & 0.679219 & & CE1 & $\mathrm{CR}$ & -0.09277 & & CE1 & CR & 0.003786 \\
\hline & & OE1 & Y3 & -0.77809 & & HE1 & H5 & 0.252083 & & HE1 & $\mathrm{H} 5$ & 0.154806 \\
\hline & & OE2 & $\mathrm{O} 2$ & -0.60672 & & NE2 & Y1 & -0.2363 & & NE2 & Y2 & -0.34032 \\
\hline & & C & C & 0.5366 & & $\mathrm{CD} 2$ & $\mathrm{CV}$ & -0.03332 & & $\mathrm{CD} 2$ & $\mathrm{CV}$ & -0.04986 \\
\hline & & $\mathrm{O}$ & $\mathrm{O}$ & -0.5819 & & HD2 & $\mathrm{H} 4$ & 0.101368 & & HD2 & $\mathrm{H} 4$ & 0.108994 \\
\hline & & & & & & $C$ & C & 0.5973 & & $\mathrm{C}$ & C & 0.5973 \\
\hline & $\begin{array}{l}\text { ZN1 } \\
727\end{array}$ & ZN & M1 & 1.037579 & & 0 & O & -0.5679 & & O & 0 & -0.5679 \\
\hline \multirow{17}{*}{ 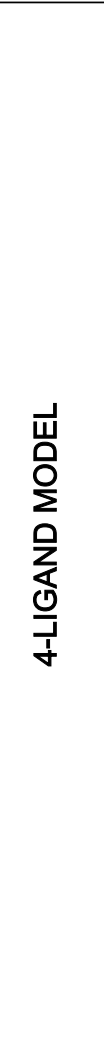 } & $\begin{array}{c}\text { GU1 } \\
508\end{array}$ & $N$ & N & -0.5163 & $\begin{array}{c}\text { HA1 } \\
450 / \\
\text { HA2 } 455\end{array}$ & $N$ & $N$ & -0.4157 & \multirow[t]{17}{*}{$\begin{array}{l}\text { ZN1 } \\
727\end{array}$} & \multirow[t]{17}{*}{ ZN } & \multirow[t]{17}{*}{ M1 } & \multirow[t]{17}{*}{1.254941} \\
\hline & & $\mathrm{H}$ & $\mathrm{H}$ & 0.370739 & & $\mathrm{H}$ & $\mathrm{H}$ & 0.34729 & & & & \\
\hline & & $\mathrm{CA}$ & $c x$ & 0.0397 & & CA & $c x$ & 0.0188 & & & & \\
\hline & & HA & $\mathrm{H} 1$ & 0.083339 & & $\mathrm{HA}$ & $\mathrm{H} 1$ & 0.140318 & & & & \\
\hline & & $\mathrm{CB}$ & $2 \mathrm{C}$ & 0.268061 & & $\mathrm{CB}$ & СT & -0.30435 & & & & \\
\hline & & HB2 & $\mathrm{HC}$ & -0.06964 & & HB2 & $\mathrm{HC}$ & 0.128156 & & & & \\
\hline & & HB3 & $\mathrm{HC}$ & -0.06964 & & HB3 & $\mathrm{HC}$ & 0.128156 & & & & \\
\hline & & $C G$ & $2 \mathrm{C}$ & -0.10473 & & CG & CC & -0.05498 & & & & \\
\hline & & HG2 & $\mathrm{HC}$ & 0.05206 & & ND1 & NA & 0.037206 & & & & \\
\hline & & HG3 & $\mathrm{Hc}$ & 0.05206 & & HD1 & $\mathrm{H}$ & 0.295424 & & & & \\
\hline & & $C D$ & $\mathrm{CO}$ & 0.697244 & & CE1 & $\mathrm{CR}$ & -0.16763 & & & & \\
\hline & & OE1 & Y3 & -0.83666 & & HE1 & H5 & 0.231563 & & & & \\
\hline & & OE2 & $\mathrm{O} 2$ & -0.63845 & & NE2 & $\mathrm{Y} 1 / \mathrm{Y} 2$ & -0.31523 & & & & \\
\hline & & C & C & 0.5366 & & CD2 & $\mathrm{CV}$ & 0.037728 & & & & \\
\hline & & \multirow[t]{3}{*}{$\mathrm{O}$} & \multirow[t]{3}{*}{ O } & \multirow[t]{3}{*}{-0.5819} & & HD2 & $\mathrm{H} 4$ & 0.095115 & & & & \\
\hline & & & & & & $C$ & $C$ & 0.5973 & & & & \\
\hline & & & & & & $\mathrm{O}$ & O & -0.5679 & & & & \\
\hline$+\frac{Q}{2}$ & $\begin{array}{c}\text { GU3 } \\
508\end{array}$ & $\mathrm{~N}$ & N & -0.5163 & $\begin{array}{c}\text { HA1 } \\
450 / \\
\text { HA2 } 455\end{array}$ & $\mathrm{~N}$ & $N$ & -0.4157 & $\begin{array}{l}\text { ZN1 } \\
727\end{array}$ & ZN & M1 & 1.212445 \\
\hline
\end{tabular}




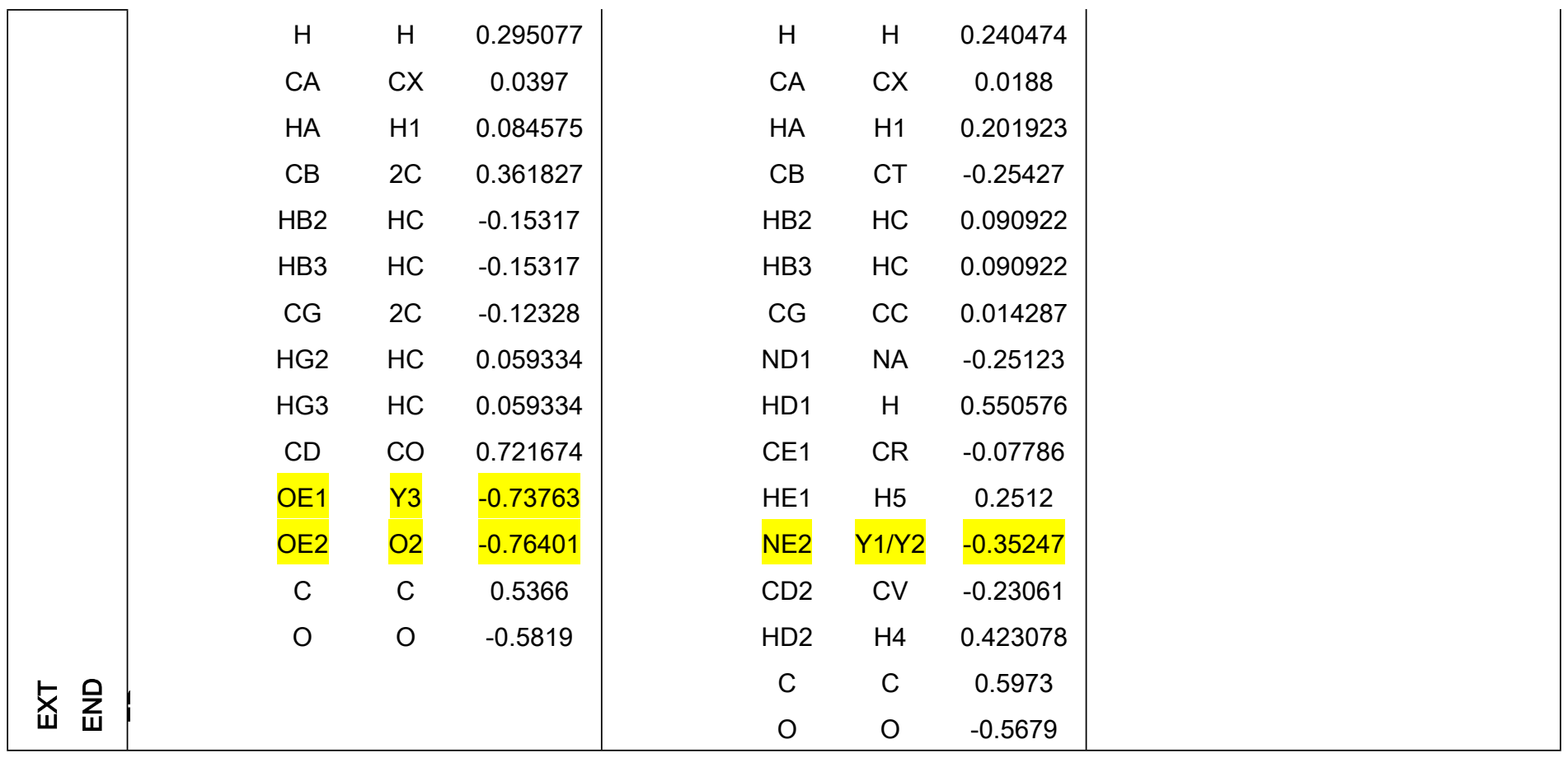

Note - It should be noted that the majority of RESP charges calculated for the first-coordination shell atoms (i.e. atoms directly participating in zinc coordination) is slightly less negative than the corresponding Amber ff12sb FF partial charges, while the zinc partial charge is in the range from 1 to 1.25. In addition, the partial atomic charges at the two E508 carboxyl oxygens, determined by the RESP procedure differ and depends on the system. 
Table S3. Results of 40-ns-long (unless otherwise specified) MD simulations of ligand-free (open, oDPP III, and closed, cDPP III) and peptide-bound (tynorphin and Leu-enkephalin, L-en) human DPP III using the ff14sb force field (unless otherwise specified) and different models to describe interactions between zinc ion and its surroundings. The boundary distance that determines whether residue coordinates the zinc ion was $2.5 \AA .<N\left(\mathrm{H}_{2} 0\right)>$ represents the average number of water molecules found within $2.5 \AA$ from the zinc ion. For each simulation the initial structure used for system setup is indicated in parenthesis.

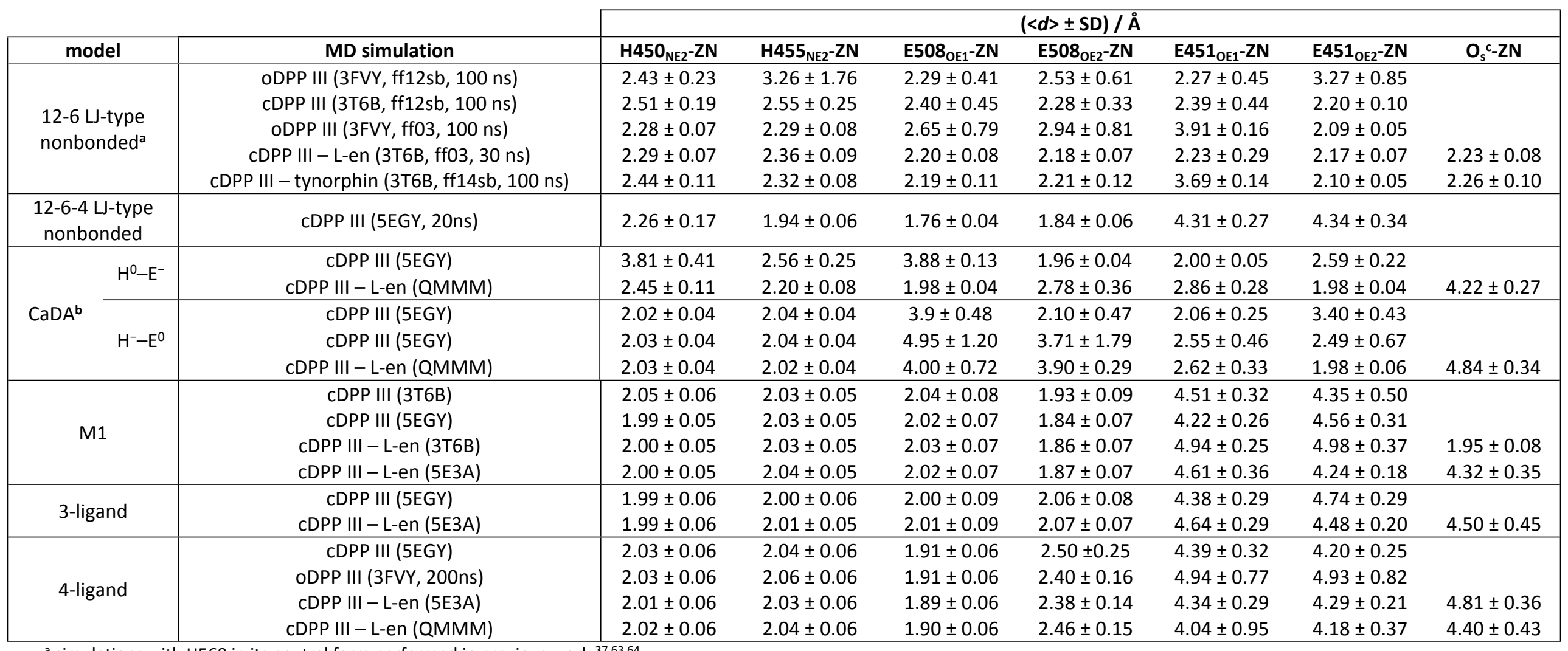

a simulations with $\mathrm{H} 568$ in its neutral form performed in previous work. ${ }^{37,63,64}$

${ }^{b}$ CaDA parameters for the zinc ion combined with parameters for: neutral $\mathrm{H} 450$ and $\mathrm{H} 455$ hydrogen bonded to negatively charged glutamates $\mathrm{E} 512$ and $\mathrm{E} 507$, respectively (marked $\mathrm{H}^{0}-\mathrm{E}^{-}$) and negatively charged $\mathrm{H} 450$ and $\mathrm{H} 455$ hydrogen bonded to neutral glutamates $\mathrm{E} 512$ and $\mathrm{E} 507$, respectively (marked $\mathrm{H}^{-}-\mathrm{E}^{0}$ ).

${ }^{c}$ average distance (and standard deviation) between the ligand $\mathrm{O}_{\mathrm{s}}$ atom (carbonyl oxygen atom from the second peptide bond from the substrate $\mathrm{N}$-terminus) and the zinc ion. 
Table S4. Hydrogen bond occupancy (\%) between substrate and protein residues calculated by Hydrogen Bonds plugin in VMD on trajectories obtained from h.DPP III - Arg-Arg-2-naphthylamide (RRNA) MD simulations. Distance and angle cut-off values of $3.0 \AA$ and $30^{\circ}$, respectively, were used. Occupancies larger than $5 \%$ are shown.

\begin{tabular}{llcc} 
donor & acceptor & $\begin{array}{c}\text { cDPP III - RRNA } \\
\text { (3T6B) }\end{array}$ & $\begin{array}{c}\text { cDPP III - RRNA } \\
(3 T 6 B)\end{array}$ \\
\hline RRNA-Side & E316-Side & 126.97 & 29.04 \\
RRNA-Main & E316-Side & 72.33 & 73.54 \\
RRNA-Side & E316-Main & & 7.26 \\
\hline RRNA-Side & S317-Main & 6.14 & \\
RRNA-Side & S317-Side & 11.20 & \\
\hline RRNA-Side & Y318-Main & & 11.96 \\
RRNA-Side & Y318-Side & 26.87 & 5.46 \\
\hline RRNA-Side & E327-Side & & 95.80 \\
\hline RRNA-Side & E329-Side & 142.37 & 109.80 \\
\hline RRNA-Side & G389-Main & 60.65 & 27.82 \\
\hline RRNA-Main & N391-Main & 10.26 & 9.80 \\
N391-Main & RRNA-Main & 45.37 & \\
\hline RRNA-Side & N394-Side & & 29.74 \\
RRNA-Side & N394-Main & 19.78 & 42.14 \\
RRNA-Main & N394-Side & 41.23 & 68.28 \\
\hline RRNA-Side & D396-Side & 45.71 & 66.40 \\
\hline RRNASide & D496-Side & 78.28 & 100.18 \\
\hline H568-Side & RRNA-Side & 64.07 & 5.58 \\
\hline
\end{tabular}


Table S5. Hydrogen bond occupancy (\%) between substrate (YGGFL) and protein residues calculated by Hydrogen Bonds plugin in VMD on trajectories obtained from h.DPP III - Leu-enkephalin MD simulations. Distance and angle cut-off values of $3.0 \AA$ and $30^{\circ}$, respectively, were used. Subscript s indicates substrate residue. Occupancies larger than $5 \%$ are shown.

\begin{tabular}{|c|c|c|c|c|}
\hline donor & acceptor & $\begin{array}{l}\text { cDPP III - L-en } \\
\text { (QMMM) }\end{array}$ & $\begin{array}{c}\text { cDPP III - L-en } \\
(5 E 3 A)\end{array}$ & $\begin{array}{c}\text { cDPP III - L-en } \\
\text { (QMMM, Drude- } \\
\text { 2013) }\end{array}$ \\
\hline $\mathrm{Y}_{\mathrm{s}}$ 1-Main & E316-Side & 19.08 & 57.46 & 29.68 \\
\hline N391-Main & $Y_{s}$ 1-Main & 20.56 & 24.66 & 25.10 \\
\hline$Y_{s} 1-$ Main & N394-Side & 47.81 & 28.00 & 18.92 \\
\hline$Y_{s} 1-$ Main & N391-Main & 59.99 & 65.74 & \\
\hline$Y_{s} 1$-Side & D496-Side & & 11.58 & \\
\hline$Y_{s}$ 1-Side & E507-Side & & & 25.90 \\
\hline $\mathrm{G}_{\mathrm{s}} 2-$ Main & E316-Side & 4.34 & & \\
\hline H568-Side & $\mathrm{G}_{\mathrm{s}} 2$-Main & 49.55 & 33.62 & \\
\hline $\mathrm{G}_{\mathrm{s}} 2$-Main & E508-Side & & & 24.10 \\
\hline G389-Main & $\mathrm{G}_{\mathrm{s}} 3$-Main & 5.90 & 78.80 & 19.92 \\
\hline $\mathrm{G}_{\mathrm{s}} 3$-Main & G389-Main & 41.09 & 50.90 & 9.76 \\
\hline A388-Main & $\mathrm{G}_{\mathrm{s}} 3$-Main & 72.95 & & 6.77 \\
\hline R572-Side & $\mathrm{F}_{\mathrm{s}} 3$-Main & 62.89 & 37.94 & 21.12 \\
\hline R669-Side & $\mathrm{F}_{\mathrm{s}}$ 3-Main & & 7.30 & \\
\hline R572-Side & $L_{s}$-Main & 9.00 & 51.00 & \\
\hline R669-Side & $\mathrm{L}_{\mathrm{s}} 4$-Side & 26.81 & 65.84 & \\
\hline R572-Side & $\mathrm{L}_{s} 4$-Side & 25.09 & 23.10 & \\
\hline R669-Side & $\mathrm{L}_{s} 4-$ Main & 68.13 & & \\
\hline
\end{tabular}




\section{The 4-ligand-extended model parameters}

The 4-ligand-extended model parameters obtained by MCPB.py program as implemented in AMBER16. Parameters are show in a frcmod file format, i.e. in an AMBER modified force field parameter file format. Corresponding atom types and charges are given in Table S1. The MCPB.py assigns zero torsion barriers for dihedral angles.

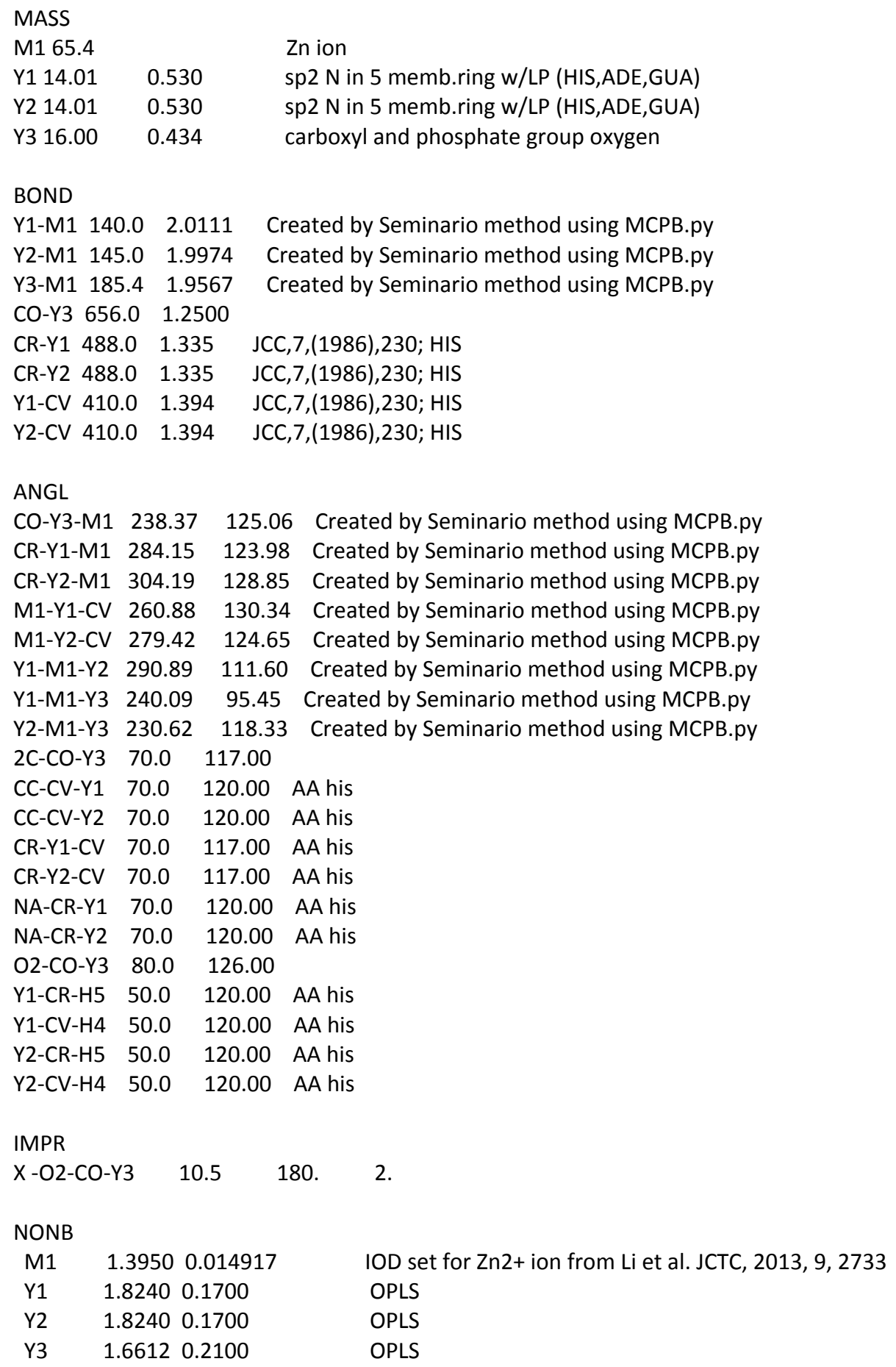




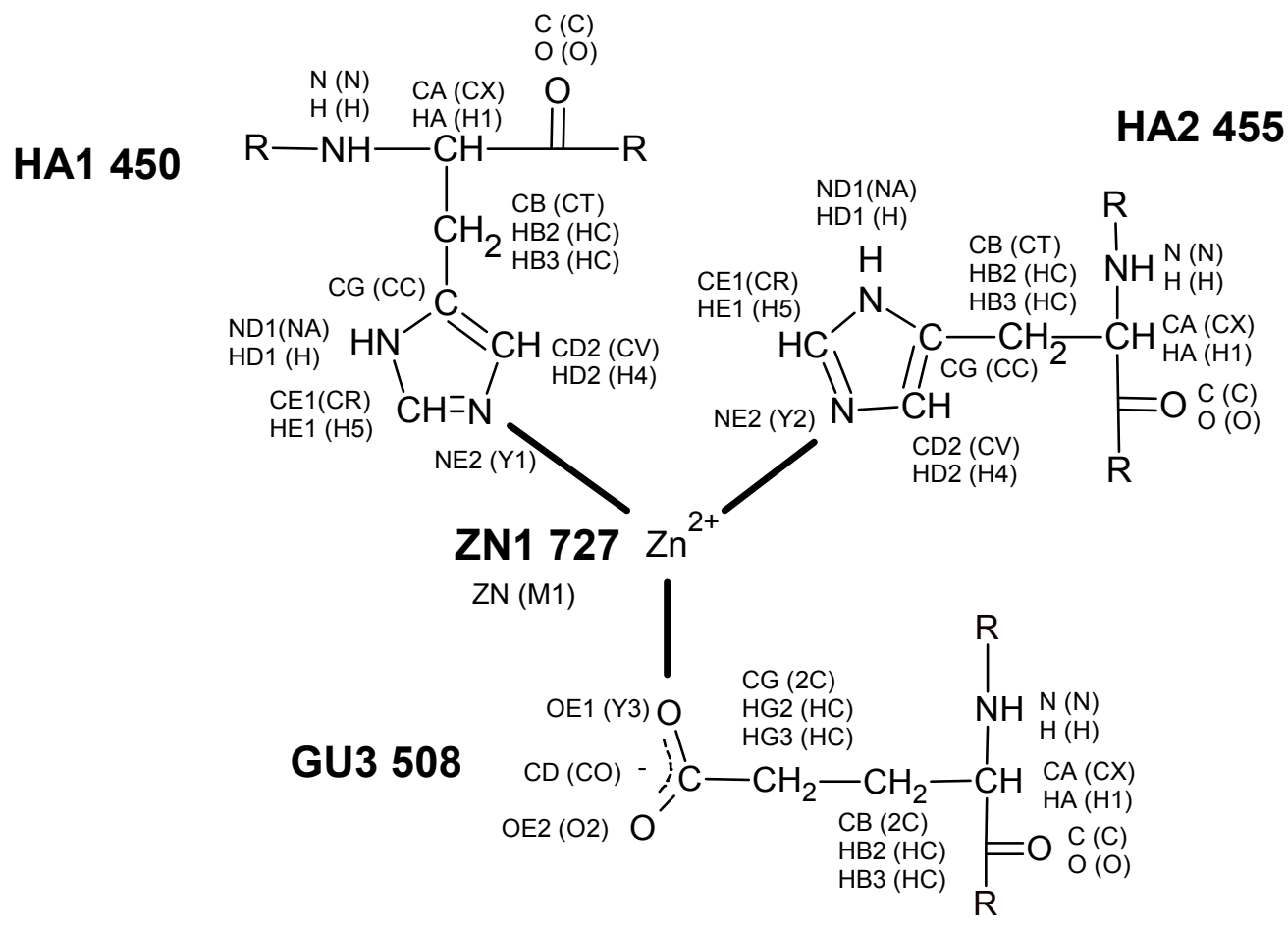

The new amino acid residues names (bold) defined by 4-ligand-extended model. Corresponding atom names and types (in paranthesis) are indicated in smaller letters. 